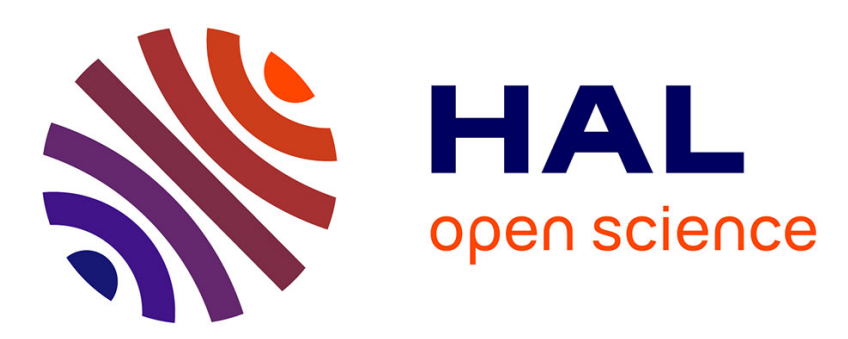

\title{
Initiation and propagation of complex 3D networks of cracks in heterogeneous quasi-brittle materials: direct comparison between in situ testing-microCT experiments and phase field simulations
}

Thanh Tung Nguyen, Julien Yvonnet, Michel Bornert, Camille Chateau

\section{To cite this version:}

Thanh Tung Nguyen, Julien Yvonnet, Michel Bornert, Camille Chateau. Initiation and propagation of complex 3D networks of cracks in heterogeneous quasi-brittle materials: direct comparison between in situ testing-microCT experiments and phase field simulations. Journal of the Mechanics and Physics of Solids, 2016, 95, pp.320-350. 10.1016/j.jmps.2016.06.004 . hal-01331213

\author{
HAL Id: hal-01331213 \\ https://hal.science/hal-01331213
}

Submitted on 13 Jun 2016

HAL is a multi-disciplinary open access archive for the deposit and dissemination of scientific research documents, whether they are published or not. The documents may come from teaching and research institutions in France or abroad, or from public or private research centers.
L'archive ouverte pluridisciplinaire HAL, est destinée au dépôt et à la diffusion de documents scientifiques de niveau recherche, publiés ou non, émanant des établissements d'enseignement et de recherche français ou étrangers, des laboratoires publics ou privés. 


\title{
Initiation and propagation of complex 3D networks of cracks in heterogeneous quasi-brittle materials: direct comparison between in situ testing- microCT experiments and phase field simulations
}

\author{
T.T. Nguyen ${ }^{\mathrm{a}, \mathrm{b}}$, J. Yvonnet ${ }^{\mathrm{a}, *}$, M. Bornert $^{\mathrm{b}}{ }^{\text {, C. Chateau }}{ }^{\mathrm{b}}$ \\ ${ }^{a}$ Université Paris-Est, Laboratoire Modélisation et Simulation Multi Échelle \\ MSME UMR 8208 CNRS, 5 bd Descartes, 77454 Marne-la-Vallée, France. \\ ${ }^{\mathrm{b}}$ Université Paris-Est, Laboratoire Navier, CNRS UMR 8205, ENPC, IFSTTAR, \\ 6/8 avenue Blaise Pascal, 77455 Marne-la-Vallée, France.
}

\begin{abstract}
We provide the first direct comparisons, to our knowledge, of complex 3D micro cracking initiation and propagation in heterogeneous quasi-brittle materials modelled by the phase field numerical method and observed in X-ray microtomography images recorded during in situ mechanical testing. Some material parameters of the damage model, including the process zone (internal) length, are identified by an inverse approach combining experimental data and $3 \mathrm{D}$ simulations. A new technique is developed to study the micro cracking at a finer scale by prescribing the local displacements measured by digital volume correlation over the boundary of a small sub-volume inside the sample during the numerical simulations. The comparisons, performed on several samples of lightweight plaster and concrete, show a remarkable quantitative agreement between the 3D crack morphology obtained by the model and by the experiments, without any a priori knowledge about the location of the initiation of the cracks in the numerical model. The results indicate that the crack paths can be predicted in a fully deterministic way in spite of the highly random geometry of the microstructure and the brittle nature of its constituents.
\end{abstract}

Key words: Micro cracking, quasi-brittle, heterogeneous material, phase-field method, gradient damage model, voxel-based models, in situ testing, X-ray microtomography, digital volume correlation

\footnotetext{
* Correspondance to J. Yvonnet

Email address: julien.yvonnet@univ-paris-est.fr (J. Yvonnet ).
} 


\section{Introduction}

Predicting the strength due to cracking in quasi-brittle materials such as concrete or other civil engineering materials is of formidable interest. Crack propagation modelling in brittle or quasi-brittle materials has long been restricted to simple academic cases and to the macroscopic scale. With recent advances in both numerical simulation methods and experimental characterization, new studies are now possible, allowing the development of fracture models at microscale, in particular from realistic microstructure morphologies of complex materials such as concrete. However, developing predictive models of crack initiation and propagation in three-dimensional complex heterogeneous microstructures is still highly challenging regarding modelling, numerical simulation methods, and experimental characterization.

Macroscopic characterization of cracks with experiments has been extensively developed in 2D and 3D for homogeneous materials. Aiming at investigating microscopic mechanisms of fracture, X-ray computed tomography (XRCT) has been used to observe in situ the 3D damage evolution of ductile materials (see e.g. Wong et al. (2006), Maire et al. (2011), Landron et al. (2011)). Nouailletas et al. (2015) investigated the effects of crack closure and opening by classical measurements and digital image correlation (DIC) in notched specimens subjected to traction/compression tests. Ferrié et al. (2005) and Toda et al. (2004) used X-ray-microtomography (XR- $\mu \mathrm{CT}$ ) to analyse 3D crack growth with closure in cast alloys at grain scale.

More recently, studies combining advanced experimental crack characterization and numerical methods have been proposed. Toshiyuki et al. (2014) studied the 3D evolution of ductile fractures and void evolutions in a thin sheet of metal by in situ testing during synchrotron-radiation computed laminography (SCRL). In this work, the comparisons with finite element computations were only performed regarding the evolution of voids. Rannou et al. (2010) and Lachambre et al. (2015) studied 3D fatigue crack growth in cast iron and used synchrotron XR- $\mu \mathrm{CT}$ combined with in situ experiments to characterize the actual geometry of the 3D crack. This geometry was used as an input in XFEM (Moës et al. (1999)) simulations to estimate the Stress Intensity Factors at the crack front. In the mentioned works, no direct comparisons between simulations and experimental cracks were performed.

Direct comparisons between experimental characterization of crack evolutions and simulations can be found in the 2D context (Ismonov and Daniewicz (2010); Oguni et al. (2009)), mainly in thin/thick plates with defects (holes or notches), where cracks are usually followed by digital image correlation (DIC) techniques. Grégoire et al. (2007) performed direct comparisons of dynamic crack paths under mixed modes in transparent PMMA loaded in split 
Hopkinson-Kolsky bars with XFEM numerical simulations. Roux-Langlois et al. (2015) analysed the displacement field with DIC on a sub-volume of the sample, to first, project the measured displacements on a Williams basis to evaluate the Stress intensity Factors of a fatigue crack, and second, to provide boundary conditions for an XFEM simulation on a sub-volume surrounding the crack. Finally, Nguyen et al. (2016a) performed direct quantitative predictions of crack propagation in drilled plaster specimen under compression with phase field numerical simulations which favourably compared with experimental investigations obtained in Romani et al. (2015).

These studies mainly deal with homogeneous samples, or with a single macro crack propagating through a macroscopically homogeneous material. The complex propagation of micro cracks or even of a network of micro cracks within a heterogeneous microstructure has been considered in other studies, but mostly only in 2D. For instance, qualitative comparisons of 2D crack propagation during shrinkage between simulation and experiments using DIC in thick plates with cylindrical heterogeneities have been proposed in Lagier et al. (2011). Comparisons between experiments and numerical simulations of 3D cracking in heterogeneous asphalt models were performed in Yin et al. (2014, 2015), but they were only qualitative. To our best knowledge, direct quantitative comparisons in 3D between numerical models of crack initiation/propagation and experimental characterization in heterogeneous media are not available so far.

The objective of the present work is precisely to tackle the difficult issue of developing a predictive model for $3 \mathrm{D}$ crack propagation in heterogeneous quasibrittle materials microstructures. We focus here on cracking in lightweight plaster and lightweight concrete microstructures. A phase field method based on the variational formulation of the crack evolution problem due to Francfort and Marigo (1998) and on its numerical implementation as proposed by Miehe et al. (2010) is used to simulate crack initiation and propagation in realistic microstructure models obtained by XR- $\mu \mathrm{CT}$. Then, in situ testing combined with $\mathrm{XR}-\mu \mathrm{CT}$ is carried out to experimentally follow the 3D crack paths by means of a specific image subtraction procedure using Digital Volume Correlation (DVC). Direct comparisons between the numerical model simulations of crack paths and experimental data are performed. In addition, some parameters of the constitutive relation of the quasi-brittle material model are identified by inverse analysis combining experiments and simulations.

Following this introduction, the experimental procedure is described in section 2. Then, the model used for quasi-brittle crack initiation and propagation based on the phase field method is presented in section 3. The identification procedure of material parameters is described in section 4. Finally, direct comparisons between the model and the experiments are provided in section 6 for lightweight plaster and lightweight concrete microstructures. Numerical 


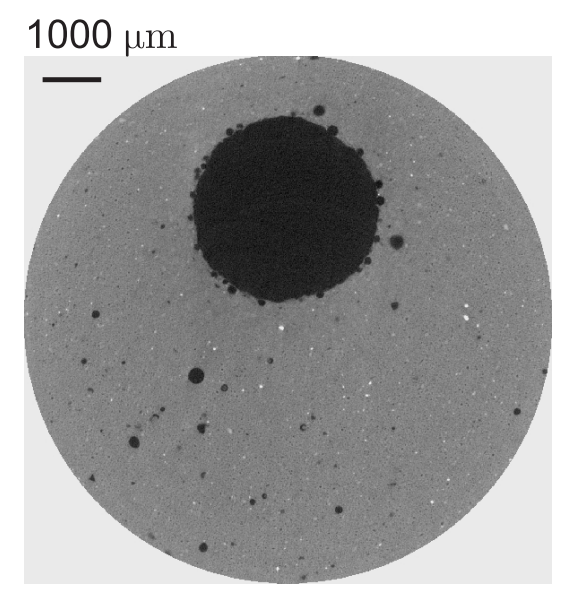

(a)

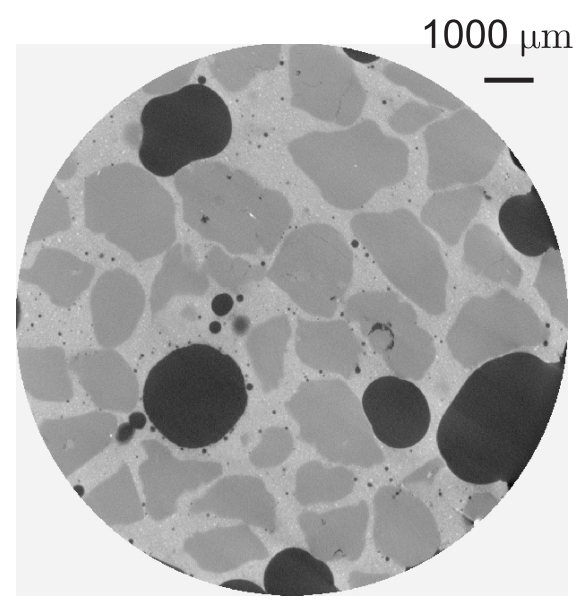

(b)

Fig. 1. Microtomography images (cross-sections) of: (a) lightweight plaster; (b) lightweight concrete.

models of the whole sample are considered in the first case, while a sub-volume technique is used in the second one, the technical details of which are provided in section 4.

\section{Experiments}

\subsection{Samples}

In the present work, two materials, lightweight plaster and lightweight concrete, are investigated. By "lightweight", we mean that the material contains very light inclusions with weak mechanical properties, here EPS (expanded polystyrene) beads, or pores (see Fig. 1). The introduction of these beads into a homogeneous plaster specimen induces first stress concentration when the sample is loaded and then crack initiation. These two materials have different microstructures (see Fig. 1. The lightweight plaster is essentially made of two phases: the plaster and the large EPS beads (pores). The pores are almost spherical, which allows an easiest modelling of the microstructure. On the other hand, the lightweight concrete contains 3 phases: the EPS beads, which play the role of pores, the matrix (cement paste) and the inclusions (sand grains with somewhat angular shapes), leading to a much more complex geometry. Note that both samples exhibit also some small-scale heterogeneities within the matrix, in the form of micro pores or small areas with higher densities (which appear in white in Fig. 1 (a)). These details are useful to run digital volume correlation (DVC) procedures but they will not be taken into account in the numerical simulations (with the exception of some larger small pores). 


\subsubsection{Lightweight plaster samples}

Plaster material is widely spread in civil engineering and its manufacturing is easy and fast. Significant research activity on this material has been reported in the literature, especially on its mechanical properties and its damage mechanisms (see e.g. Saadaoui et al. (2000); Meille et al. (2003); Janeiro and Einstein (2010); Romani (2013); Romani et al. (2015)). The specific plaster used in this study is the same as the one used by Romani et al. (2015), manufactured by the Siniat company (commercial reference Prestia Profilia 35(R)). Plaster exhibits a brittle behaviour. In order to develop a stable crack network, and localize it in a specific region of the sample, EPS beads have been incorporated within the plaster matrix. EPS stiffness is very low (4-8 MPa) so that the beads can be assimilated to pores. Lightweight plaster is thus composed of two phases: the assumed homogeneous plaster matrix and porosity, comprising the incorporated EPS beads which are millimetric in size but also some additional smaller pores induced by the manufacturing process, which can be observed in Fig. 1 (a). Specific experimental procedures have been developed to manufacture small EPS plaster samples with controlled microstructure and overall geometry, adapted to in situ compression testing combined with XR$\mu \mathrm{CT}$ imaging. (see Nguyen (2015) for more details). The following presentations and discussions will be focused on two specific EPS plaster specimens referred to as "PlasterS1" and "PlasterS2" samples, each containing two EPS beads with a diameter of about 3 or $4 \mathrm{~mm}$. Detailed information about both specimens is provided in Table 1 .

\subsubsection{Lightweight concrete samples}

Lightweight concrete (or EPS concrete) includes quartz sand and EPS beads embedded in a cement matrix. This material is a multi-purpose material for construction, and offers a large range of enhanced technical and economical features. EPS concrete has some distinguished advantages as compared to classical concrete, like higher strength to weight ratio, better tensile strain capacity with respect to classical concrete, and superior heat and sound insulation characteristics due to inclusion of air voids in the lightweight aggregate. However, the introduction of the EPS beads reduces the strength, and the study of micro cracking in such materials is thus critical for civil engineering applications. The size effect of polystyrene aggregates on strength and moisture migration characteristics of lightweight concrete was analysed by Babu et al. (2006) and on the compressive strength by Liu and Chen (2014). The fracture phenomena of this typical material at macro scale have mainly been studied under compression by Miled et al. (2004, 2007). The XR- $\mu$ CT technique has been applied to study such material in Sprague (2006) as well as in Lu et al. (2006). 


\begin{tabular}{|c|c|c|c|c|c|}
\hline Specimen & $\begin{array}{c}\text { Height } \\
(\mathrm{mm})\end{array}$ & $\begin{array}{c}\text { Diameter } \\
(\mathrm{mm})\end{array}$ & $\begin{array}{c}\text { Mass } \\
(\mathrm{g})\end{array}$ & Beads nb. & $\begin{array}{c}\text { Porosity } \\
(\%)\end{array}$ \\
\hline PlasterS1 & 18.2 & 9.0 & 2.6 & 2 (about 3 mm in diameter) & 4.3 \\
PlasterS2 & 18.2 & 9.0 & 2.5 & 2 (about 4 mm in diameter) & 5.3 \\
ConcreteS1 & 19.9 & 11.7 & 2.3 & Multiple (about 1 to 2 mm in diameter) & 22.0 \\
\hline
\end{tabular}

Table 1

Information about observed specimens

Several samples of EPS concrete were provided by Prof K. Miled (ENIT, Tunis). A cylindrical specimen (11.7 $\mathrm{mm}$ in diameter and $19.9 \mathrm{~mm}$ long) was machined using diamond core drilling and cutting from a cubic sample. Details about the obtained specimen (referred to as "ConcreteS1") are given in Table 1. A slice of a XR- $\mu \mathrm{CT}$ image showing the microstructure is provided in Fig. 1(b). The typical sizes of the components are about $1 \mathrm{~mm}$ for sand grains and $2 \mathrm{~mm}$ for EPS beads. In addition to the EPS beads, the observed porosity also contains air voids of various smaller sizes. Using a segmentation of the CT image of the whole specimen, the volume fractions have been estimated to about $22 \%$ for porosity (EPS beads and residual porosity) and $57 \%$ for quartz sand.

\subsection{Description of the in situ compression test}

The aim of the experiments is to study in $3 \mathrm{D}$ the evolution of the network of micro cracks at several compressive loading levels, by means of XR- $\mu \mathrm{CT}$ imaging. To do so, a specific in situ compression device has been used, which allows to prescribe a constant loading state to the sample during each $\mu \mathrm{CT}$ scan. Such a procedure is preferred to a simpler ex situ protocol, during which the sample would be unloaded for the scans, which might induce a closure of the cracks and a much less efficient detection.

The tests were performed on the XR- $\mu \mathrm{CT}$ laboratory scanner available at Laboratoire Navier (Ultratom from RX-Solution), specifically designed to receive in situ experiments. The compression in situ machine used for this study has been designed and manufactured by LMS (Ecole polytechnique). A view of the in situ experiment performed on the laboratory tomograph is depicted in Fig. 3. The in situ device is mainly made of steel to ensure the mechanical strength. The transparency to X-rays is provided by a polycarbonate tube around the specimen (see Fig. 2(a)). The specimen is centred in the beam with alignment screws at the bottom of the machine. The loading capacity of $10 \mathrm{kN}$ is sufficient to load the different specimens until rupture (up to about $3 \mathrm{kN}$ ). Very low displacement rates (down to $0.1 \mu \mathrm{m} / \mathrm{s}$ ) can be applied to ensure a slow propagation of cracks. 


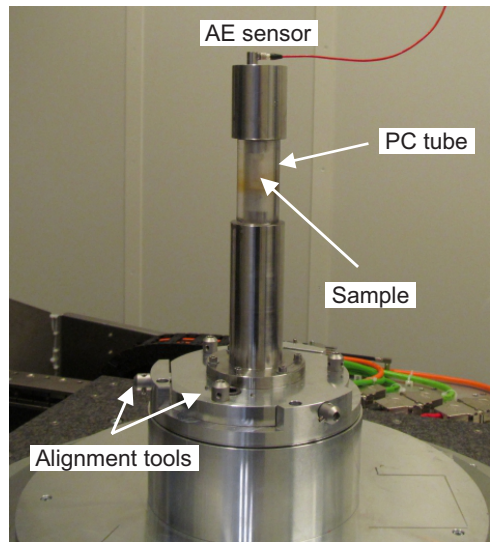

(a)

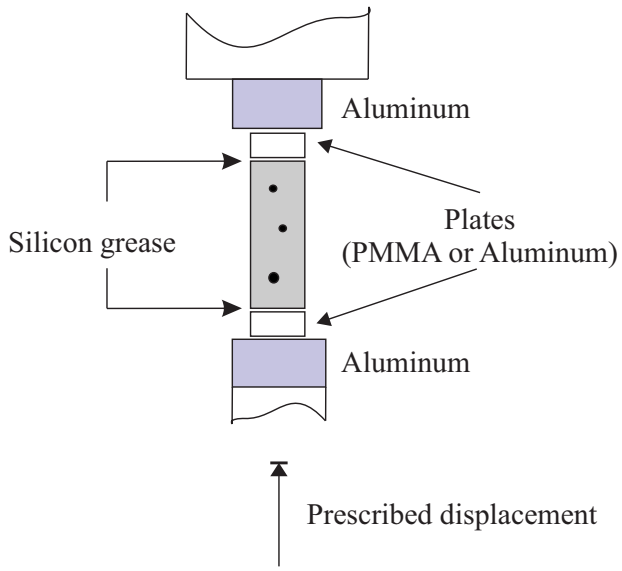

(b)

Fig. 2. (a) In situ compression testing machine and acoustic emission monitoring; (b) Details of the setup for the application of the uniaxial compression load on the EPS concrete sample.

The load has been prescribed to the specimen through aluminium rods with low X-ray attenuation to limit imaging artifacts at the boundaries. Special care is in addition taken to centre the specimen on these rods and on the machine. As described in Fig. 2 (b), two PMMA or aluminium plates with same diameter as the sample are in addition placed on top and bottom of the sample. Indeed, as described in more details in Nguyen (2015), the transverse strain prescribed by the plates to the sample at its ends plays a central role on the initiation of damage near the ends of the sample. It depends on the discrepancy of the Poisson's ratio/Young's modulus ratios of the materials in contact and on the friction coefficient. Several solutions have been used to reduce this effect: (1) the plates have been machined with the same cylindrical shape and the same diameter as the sample to avoid the punching effect; (2) they have been carefully polished at their interface with the sample; (3) some silicon grease has been added at the contact regions between sample and plates in order to reduce friction effects. The PMMA plates have been used for the test on sample PlasterS1 and the aluminium plates for the samples PlasterS2 and ConcreteS1.

Several successive loading steps have been applied to each specimen until failure. Details about the loading of each specimen are presented in Table 2, including the step at which the cracks were first detected. Note that the compression tests of lightweight plaster specimens are composed of several loading phases, between which the samples were partially unloaded during nights (each test lasted several days). The whole test of the lightweight concrete has been carried out in only one loading phase, without intermediate unloading. Note that at least two very small and stable cracks were noticed from $F=0.94 \mathrm{kN}$. Nevertheless, the crack network significantly propagates only from $\mathrm{F}=1.454$ $\mathrm{kN}$, which is considered as the crack initiation loading in the present study 


\begin{tabular}{|c|c|c|c|c|}
\hline Name & $\begin{array}{c}\text { Number of loading } \\
\text { phases }\end{array}$ & $\begin{array}{c}\text { Number of loading } \\
\text { steps }\end{array}$ & $\begin{array}{c}F_{\mathrm{i}} \\
(\mathrm{kN})\end{array}$ & $\begin{array}{c}F_{\max } \\
(\mathrm{kN})\end{array}$ \\
\hline PlasterS1 & 6 & 19 & 2.76 & 2.88 \\
\hline PlasterS2 & 3 & 15 & 1.62 & 2.71 \\
\hline ConcreteS1 & 1 & 12 & 1.45 & $\ldots$ \\
\hline
\end{tabular}

Table 2

Summary of performed in situ compression tests

The macroscopic compression responses of the three specimens are presented in Fig. 4. In these plots, the overall load is measured by the loading sensor of the in situ compression device, while the strains are measured by Digital Volume Correlation (DVC) techniques, as described hereafter.

It is noted that no softening regime could be observed during the experiments. A reason for that is that the compression device is not sufficiently rigid to control accurately the displacement prescribed to the sample. Indeed the PC tube required for the X-ray imaging, together with the global geometry of the loading device which needs to be rather long to allow the X-ray source to get close to the sample, induce some compliance to the testing device. In the softening regime of the sample, the elastic energy stored in the device is transferred to the sample which generates a global instability leading to the full dynamic failure of the sample. In addition, there are probably also local instabilities, which are very difficult to control and detect. However, as depicted in Fig. 4, the first cracks appear way before the global rupture of the specimen, so that experimental progressive evolution of the crack network can be compared to simulation for a significant loading history.

In Table $2, F_{\mathrm{i}}$ is the prescribed force of the first crack loading step and $F_{\max }$ is the rupture force. From Fig. 4, we can note that the macroscopic response of the two plaster samples are similar, and their behaviour in the elastic regime are comparable. However, the first cracks in PlasterS2 sample have been detected at $F_{i}=1.62 \mathrm{kN}$, against $F_{i}=2.76 \mathrm{kN}$ for the PlasterS1 sample. This can be understood from the larger porosity of the first specimen, where in addition the large pores and beads are located near the boundaries, inducing weaker zones. The ConcreteS1 sample exhibits a different behaviour. The maximal longitudinal strain is about $10^{-3}$, against $3-4 \times 10^{-3}$ for the plaster samples. This is because the lightweight concrete is stiffer but contains much more pores than the plaster samples; its maximal load is lower (about $2 \mathrm{kN}$ i.e. $19 \mathrm{MPa}$ ) than that of the plaster samples (about 2.5 to $3 \mathrm{kN}$, i.e. 39 to $47 \mathrm{MPa}$ ). This might be linked to the fact that despite the stiffer behaviour of the cement paste and the sand grains in the concrete, with respect to the plaster matrix, the much larger porosity of the concrete sample induces damage at a lower overall load. In addition, it can be observed that the transition 


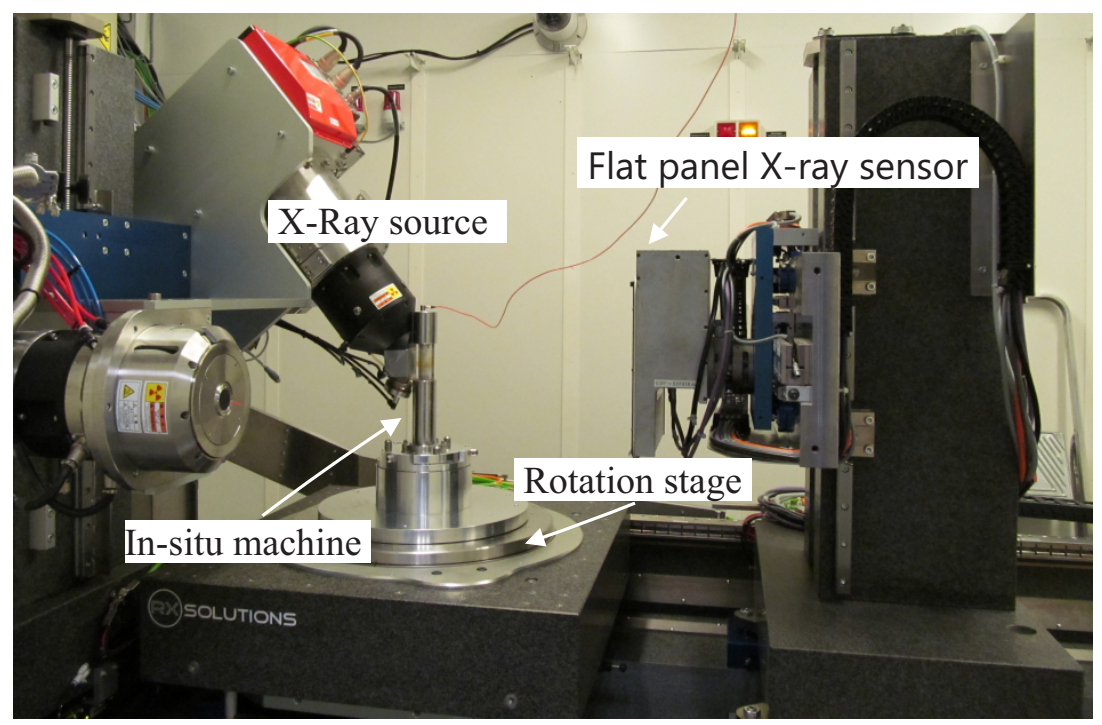

Fig. 3. Global view of the in situ compression test combined with XR- $\mu \mathrm{CT}$ at Laboratoire Navier.

between the purely elastic behaviour and the response of the damaged sample is more abrupt for concrete, while the stiffness reduction for plaster samples due to damage apparition is limited. This might be linked to a higher density of microcracks in the concrete sample with a larger porosity and a much more complex microstructure.

\section{$2.3 \quad X R-\mu C T$ imaging and $D V C$}

The CT images of this work were captured on the XR- $\mu \mathrm{CT}$ laboratory scanner available at Laboratoire Navier (Ultratom from RX-Solution) using a microfocus source Hamamatsu L10801 (max. tension $230 \mathrm{kV}$, max. power $200 \mathrm{~W}$, minimal spot size $5 \mu \mathrm{m}$ ) and a "flat-panel" imager Paxscan Varian $2520 \mathrm{~V}$ (1920x1536 pixels, pixel size $127 \mu \mathrm{m})$. The hollow rotation stage is able to support heavy loads (up to $100 \mathrm{~kg}$ ) and was designed to receive in situ experiments. A view of the in situ experiments performed on the laboratory tomograph is depicted in Fig. 3.

$\mathrm{CT}$ images of the whole specimens were acquired under constant load at each loading step. The characteristics of 3D images are described in Table 3 for each specimen. For the PlasterS1 and PlasterS2 samples, the tube voltage and tube current have been chosen as $120 \mathrm{kV}$ and $67 \mu \mathrm{A}$, respectively, and the tube voltage and tube current have been chosen as $130 \mathrm{kV}$ and $67 \mu \mathrm{A}$ for the ConcreteS1 sample.

$\mathrm{X}$-Ray radiographs were acquired every $0.25^{\circ}$. Two $360^{\circ}$ scans (i.e. a total of 2880 projections) were necessary to cover the whole EPS plaster and EPS 


\begin{tabular}{|c|c|c|c|c|c|}
\hline Name & $\begin{array}{c}\text { Voxel size } \\
\mu \mathrm{m}\end{array}$ & $\begin{array}{c}\text { Volume size } \\
\text { Voxel }^{3}\end{array}$ & $\begin{array}{c}\text { Frame rate } \\
(\mathrm{proj} . / \mathrm{s})\end{array}$ & $\begin{array}{c}\text { Average } \\
(\text { projections })\end{array}$ & $\begin{array}{c}\text { Scan time } \\
(\mathrm{h})\end{array}$ \\
\hline PlasterS1 & 8 & $1150 \times 1150 \times 2250$ & 3 & 12 & 3 \\
\hline PlasterS2 & 8 & $1150 \times 1150 \times 2300$ & 2 & 8 & 3 \\
\hline ConcreteS1 & 10 & $1200 \times 1200 \times 2000$ & 3 & 12 & 3 \\
\hline
\end{tabular}

Table 3

Summary of X-ray tomography settings

concrete heights using a helical image acquisition mode, in which source and detector are moved vertically synchronously with the angular rotation of the sample. In order to improve signal to noise ratios of the projections, several radiographs recorded at a same angular position were averaged. The frame rate of the image sensor and the number of images averaged are indicated in Table 3. Altogether, these imaging conditions resulted is scan times of about 3 hours for a single 3D image, and the whole mechanical tests last several days. $3 \mathrm{D}$ images have been reconstructed by means of the X-Act software provided by the manufacturer of the CT device, based on the filtered back projections, and the Feldkamp et al. (1984) algorithm for conical geometry. Local DVC analyses (Bay et al. (1999)) were then performed on the images, using the inhouse software CMV_3D developed at Laboratoire Navier, see Bornert et al. (2004); Lenoir et al. (2007). The macroscopic axial strain component (or vertical strain) (see Fig. 4) is here determined from DVC data. The displacements from initial to current configuration of a set of points located on two planes near the ends of the sample are determined by DVC and used as the nodal displacements of a finite element mesh, made of 8-node elements, covering almost the whole specimen. The average strain of this mesh computed with standard integration procedures provides the overall strain of the sample (see also Lenoir et al. (2007).

\subsection{Detection of damage from DVC-assisted image subtraction and image transformation}

CT images of the last loading steps show cracks that progressively develop in both samples. However, because their grey level are very similar to those of the porosity and close to those of the various constituents, the extraction of cracks from CT images with conventional segmentation tools, especially in their early stage of initiation, is not straightforward. Therefore, a specific procedure has been developed to detect and extract microcracks from the DVC local measurements.

DVC is a direct 3D extension of Digital Image Correlation (Bay et al. (1999); Bornert et al. (2004, 2012)), requiring local image contrast to track kinematic 


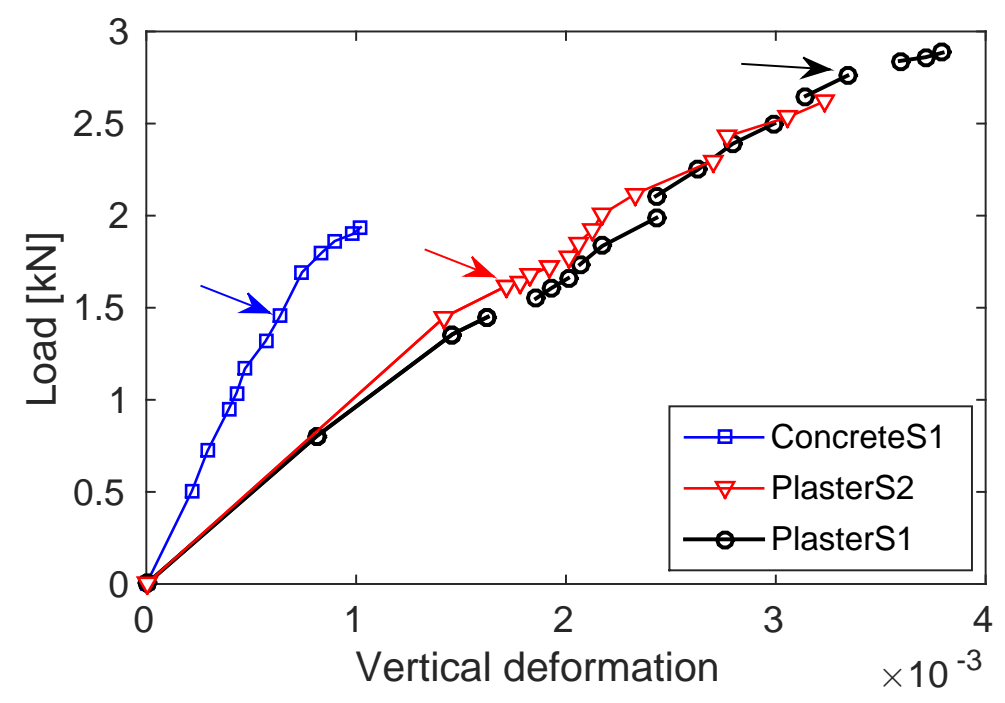

Fig. 4. Macroscopic load-strain response of the three samples obtained from in situ compression testing (arrows show the first loading step at which cracks are observed).

transformation of a sample, characterised by a map $\boldsymbol{\Phi}$. In the case of EPS plaster, the local contrast in plaster provided by microvoids and small dense inclusions (see figure 1 and section 2.1) turns out to be sufficient to run DVC routines all over the solid phase of the samples. However, in the case of EPS concrete, the grey levels in EPS beads and sand grains are rather uniform. Then, local DVC routines have been carried out in cement matrix only, and in a close neighbourhood of the sand grain interfaces.

The obtained sparse evaluation of the transformation map $\boldsymbol{\Phi}$ can be continuously extended throughout the whole sample, by means of an interpolation procedure: the transformation at any voxel in the reference image is obtained by a first order fit of the displacement of at least 4 non-coplanar neighbour positions successfully investigated by DVC. The grey level function $g(\mathbf{\Phi}(\mathbf{x}))$ in the deformed image at the final position $\mathbf{\Phi}(\mathbf{x})$ of any voxel $\mathbf{x}$ with grey level $f(\mathbf{x})$ in the reference image is obtained by a tricubic interpolation of the deformed image. The deformed image is back convected this way in the same frame as the reference image according to the estimated transformation. Finally, the difference between both reference and deformed images defines the "subtracted image" as:

$$
r(\mathbf{x})=a . g(\mathbf{\Phi}(\mathbf{x}))+b-f(\mathbf{x})+c .
$$

This definition of the image subtraction allows for a possible variation in image brightness and contrast. The coefficient $a$ reflects a possible evolution in contrast, while coefficient $b$ accounts for a brightness fluctuation. In addition, the offset $c$, taken as $c=128$ for 8-bit grey levels images, ensures a positive grey level of the obtained image. Coefficients $a$ and $b$ are determined in such 
a way that the grey level histograms of both images match. Several pairs of coefficients might be used for different areas of the images (see Nguyen (2015) for details). The final value for the case of unsigned 8 bit images is computed as Eq. (1).

The subtracted image reflects the local evolutions within the material, not described by the fit of the coarse evaluation of the transformation provided by DVC measurements and their extrapolation. For a brittle material, it essentially gives access to the cracks. Note that within areas with sufficient local contrast where DVC routines are run successfully, the subtracted image coincides with standard so-called "correlation residuals" (Bornert et al. (2012)), if $a=1$ and $b=0$. The presented procedure extends the latter approach to the current situation of a non-uniform distribution of contrast and non-perfect grey level convection.

The subtracted images essentially consist in an uniformly grey background, with an average grey level $c$, in which the crack network appears in dark grey. It can be shown that the grey level of the crack reflects their opening (Nguyen (2015)). A segmentation of this crack network is possible with basic thresholding routines, when the signal to noise ratio of the CT images is sufficiently good, i.e. when the grey level of the cracks is sufficiently different from the fluctuations of the background grey level due to image noise. This was the case for lightweight concrete samples, and the results presented in section 6 for this material have been obtained this way. More precisely, the segmented crack network could be superimposed to the segmented reference image, in which voids, sand grains and cement matric can be separated thanks to their strongly different grey levels.

For plaster samples, the signal to noise ratio of the images was less good, so that the extraction of the crack network from the subtracted image was not straightforward, and would have required more technical image filtering. Fortunately, in plaster samples, the grey level of the solid (plaster) phase is sufficiently uniform so that cracks can be qualitatively observed without the subtraction technique. In order to unambiguously follow the damage evolution during the compression tests, an alternative technique has however been used. It consists in back-convecting the CT images of the deformed sample to the reference frame. More precisely, the "transformed image" is defined as:

$$
\operatorname{Transf}(\mathbf{x})=g(\mathbf{\Phi}(\mathbf{x}))
$$

where $g(\Phi(x))$ is computed with exactly the same procedure as in Eq (1). In such an image, cracks can be observed almost as clearly as in the deformed image, with the additional advantage that any cross section of the transformed image can be directly compared to the corresponding cross section in the reference image, or in the transformed image associated to any other loading step. 
The evolution of the crack can then be unambiguously observed, which would not have been the case when reference and deformed images are compared, because in the letter case, apparent evolutions of the cracks in the observed cross-sections stem both from their physical evolution and the change of point of view. In addition, in such transformed images the microstructure of the samples (i.e. EPS beads and micropores) are still visible, so that the crack path can be compared to the microgeometry of the material. Results presented in section 6 on EPS plaster samples have been obtained this way.

\section{Modeling}

\subsection{Phase field model of regularized brittle fracture}

In the present work, crack initiation and propagation are modelled within quasi-brittle materials by means of the phase field method, whose basic concepts are reviewed in the following. For more details and practical implementation aspects, the interested reader can refer to Miehe et al. (2010); Nguyen et al. (2015). The phase field method is based on a regularized formulation of a sharp crack description. A regularized variational principle describing both the evolution of the mechanical problem and of an additional field $d$ describing the damage (called phase field), is discretized by a finite element procedure and a staggered algorithm, chosen here due to its easier implementation. The method alleviates the shortcomings of remeshing crack geometry by using a fixed mesh and a regularized description of the discontinuities. In addition, crack initiation can be modelled in a straightforward manner. In contrast to volume damage models, usually implemented in nonlinear codes, such regularized approach is directly connected to the brittle crack theory of crack propagation. In the present work, the phase field method has been implemented in a in-house code both in 2D and 3D developed at MSME lab..

The variational approach to fracture mechanics provided by Francfort and Marigo (1998) introduces the following energy functional for cracked body:

$$
E(\mathbf{u}, \Gamma)=E_{u}(\mathbf{u}, \Gamma)+E_{s}(\Gamma)=\int_{\Omega \backslash \Gamma} W_{u}(\varepsilon(\mathbf{u})) d \Omega+g_{c} \mathcal{H}^{d-1}(\Gamma)
$$

where $W_{u}$ is the energy density function, $\varepsilon=\frac{1}{2}\left(\nabla \mathbf{u}+\nabla \mathbf{u}^{T}\right)$, $\mathbf{u}$ is the displacement field, $g_{c}$ is the fracture toughness, and $\mathcal{H}^{d-1}$ is the Hausdorff surface measure giving the crack length $(d=2)$ or surface $(d=3)$. The term $E_{u}(\mathbf{u}, \Gamma)$ represents the elastic energy stored in the cracked body, and $E_{s}(\Gamma)$ is the energy required to create the crack according to the Griffith criterion. Then, the state variables are the displacement field $\mathbf{u}$ and the geometry of the 
crack $\Gamma$. In a regularized framework (phase field method), the above functional is substituted by the functional:

$$
E(\mathbf{u}, d)=\int_{\Omega} W_{u}(\varepsilon(\mathbf{u}), d) d \Omega+g_{c} \int_{\Omega} \gamma(d, \nabla d) d \Omega
$$

In $(4), \gamma(d, \nabla d)$ denotes the crack density function per unit volume, defined by:

$$
\gamma(d, \nabla d)=\frac{1}{2 \ell} d^{2}+\frac{\ell}{2} \nabla d \cdot \nabla d
$$

where $\ell$ is a parameter defining the regularized description of the cracks. Let the symbols $\nabla($.$) and \nabla \cdot($.$) denote the gradient and divergence operators,$ respectively. The total energy is then rewritten as $E=\int_{\Omega} W d \Omega$ with

$$
W=W_{u}(\varepsilon(\mathbf{u}), d)+g_{c} \gamma(d) .
$$

Assuming isothermal process, the Clausius-Duhem inequality states that:

$$
\phi=\boldsymbol{\sigma}: \dot{\boldsymbol{\varepsilon}}-\dot{W} \geq 0
$$

where $\boldsymbol{\sigma}$ is the Cauchy stress and $\phi$ is the dissipation. We can re-write (7) as:

$$
\boldsymbol{\sigma}: \dot{\boldsymbol{\varepsilon}}-\frac{\partial W}{\partial \boldsymbol{\varepsilon}}: \dot{\boldsymbol{\varepsilon}}-\frac{\partial W}{\partial d} \dot{d}=\left(\boldsymbol{\sigma}-\frac{\partial W}{\partial \boldsymbol{\varepsilon}}\right): \dot{\boldsymbol{\varepsilon}}-\frac{\partial W}{\partial d} \dot{d} \geq 0
$$

It follows that if no damage occurs, i.e. for $\dot{d}=0$, then $\phi=0$ and

$$
\sigma=\frac{\partial W}{\partial \varepsilon}
$$

A reduced form of the Clausius-Duhem inequality can be re-written as:

$$
\mathcal{A} \dot{d} \geq 0
$$

where $\mathcal{A}=-\frac{\partial W}{\partial d}$ is the thermodynamic force associated with $d$.

At this stage, a threshold function $F(\mathcal{A})$ such that no damage occurs is assumed in the form:

$$
F(\mathcal{A})=\mathcal{A} \leq 0
$$

Assuming the principle of maximum dissipation then requires the dissipation $\mathcal{A} \dot{d}$ to be maximum under the constraint (11). Using the method of Lagrange multipliers and the following Lagrangian:

$$
\mathcal{L}=-\mathcal{A} \dot{d}+\lambda F(\mathcal{A})
$$


yields the Kuhn-Tucker equations:

$$
\frac{\partial \mathcal{L}}{\partial \mathcal{A}}=0, \quad \lambda \geq 0, F \leq 0, \lambda F=0
$$

The first equality in (13) gives:

$$
\dot{d}=\lambda \frac{\partial F(\mathcal{A})}{\mathcal{A}}=\lambda
$$

Using the second inequality in (13), we obtain $\dot{d} \geq 0$. For $\dot{d}>0, F=0$ which gives:

$$
F=-\frac{\partial W}{\partial d}=-\frac{\partial W_{u}(\mathbf{u}, d)}{\partial d}-g_{c} \delta \gamma(d)=0
$$

with (see e.g. Miehe et al. (2010)):

$$
\delta \gamma(d)=\frac{d}{\ell}-\ell \Delta d
$$

where $\Delta($.$) denotes the Laplacian operator. In the present work, and following$ Miehe et al. (2010), we choose the following form for $W_{u}$, assuming isotropic elastic behavior of the body accounting for damage induced by traction only, through:

$$
W_{u}(\mathbf{u}, d)=\Psi^{+}(\varepsilon(\mathbf{u}))\{g(d)+k\}+\Psi^{-}(\varepsilon(\mathbf{u})) .
$$

The strain field is decomposed into extensive and compressive modes as

$$
\varepsilon=\varepsilon^{+}+\varepsilon^{-}
$$

and

$$
\begin{aligned}
& \Psi^{+}(\varepsilon)=\frac{\lambda}{2}\left(\langle\operatorname{Tr}(\varepsilon)\rangle_{+}\right)^{2}+\mu \operatorname{Tr}\left\{\left(\varepsilon^{+}\right)^{2}\right\} \\
& \Psi^{-}(\varepsilon)=\frac{\lambda}{2}\left(\langle\operatorname{Tr}(\varepsilon)\rangle_{-}\right)^{2}+\mu \operatorname{Tr}\left\{\left(\varepsilon^{-}\right)^{2}\right\}
\end{aligned}
$$

where

$$
\boldsymbol{\varepsilon}^{+}=\sum_{i=1}^{D}\left\langle\varepsilon^{i}\right\rangle_{+} \mathbf{n}^{i} \otimes \mathbf{n}^{i}, \quad \boldsymbol{\varepsilon}^{-}=\sum_{i=1}^{D}\left\langle\varepsilon^{i}\right\rangle_{-} \mathbf{n}^{i} \otimes \mathbf{n}^{i} .
$$

where $\varepsilon^{i}$ and $\mathbf{n}^{i}$ are the eigenvalues and eigenvectors of $\boldsymbol{\varepsilon}$, i.e. satisfying $\boldsymbol{\varepsilon} \mathbf{n}^{i}=$ $\varepsilon^{i} \mathbf{n}^{i}$. In $(21)\langle x\rangle_{+}=(x+|x|) / 2$ and $\langle x\rangle_{-}=(x-|x|) / 2$. The degradation 
function $g(d)$ in Eq. (17) is assumed to have the simple form:

$$
g(d)=(1-d)^{2}
$$

The function $g(d)$ has been chosen such that $g^{\prime}(1)=0$ to guarantee that the strain energy density function takes a finite value as the domain is locally cracked (see e.g. Braides (1998)), and $g(0)=1$ to guarantee that the material is initially undamaged, and $g(1)=0$ for full damage. The power 2 is the smallest power to guarantee the existence of a solution in the sense of Carfagni.

The small parameter $k<<1$ is introduced to maintain the well-posedness of the system for partially broken parts of the domain (Miehe et al. (2010)). It follows that if $\dot{d}>0$ then:

$$
\mathcal{A}=-\frac{\partial W}{\partial d}=2(1-d) \Psi^{+}-g_{c} \delta \gamma(d)
$$

As $2(1-d) \Psi^{+} \geq 0$, then

$$
\delta \gamma(d) \geq 0, \quad \text { if } \dot{d}>0
$$

Expressing the variation of crack length:

$$
\dot{\Gamma}_{\ell}=\int_{\Omega} \delta \gamma(d) \dot{d} d \Omega
$$

we can check that due to $(24)$

$$
\dot{\Gamma}_{\ell} \geq 0
$$

satisfying non-reversible evolution of cracks.

In addition, to handle loading and unloading histories, Miehe et al. (2010) introduced the strain history functional:

$$
\mathcal{H}(\mathbf{x}, t)=\max _{\tau \in[0, t]}\left\{\Psi^{+}(\mathbf{x}, \tau)\right\}
$$

which is substituted to $\Psi^{+}$in (15). For a discussion and justification of the use of this function, the reader is invited to refer to the mentioned reference. It yields the following phase field problem to be solved to evaluate the field $d(\mathbf{x}, t)$ at time $t$ :

$$
\left\{\begin{array}{l}
2(1-d) \mathcal{H}-\frac{g_{c}}{\ell}\left\{d-\ell^{2} \Delta d\right\}=0 \text { in } \Omega \\
d(\mathbf{x})=1 \text { on } \Gamma \\
\nabla d(\mathbf{x}) \cdot \mathbf{n}=0 \text { on } \partial \Omega
\end{array}\right.
$$


The Euler-Lagrange equations of the variational principle

$$
\mathbf{u}(\mathbf{x})=\operatorname{Arg}\left\{\inf _{\mathbf{u} \in \mathcal{S}_{u}}\left(E(\mathbf{u}, d)-W^{e x t}\right)\right\}
$$

where $\mathcal{S}_{u}=\left\{\mathbf{u} \mid \mathbf{u}(\mathbf{x})=\overline{\mathbf{u}}\right.$ on $\left.\partial \Omega_{u}, \quad \mathbf{u} \in H^{1}(\Omega)\right\}$ and $W^{\text {ext }}=\int_{\Omega} \mathbf{f} \cdot \mathbf{u} d \Omega+\int_{\partial \Omega_{F}} \overline{\mathbf{F}}$. $\mathbf{u} d \Gamma$ with $\mathbf{f}$ and $\overline{\mathbf{F}}$ body forces and prescribed traction over the boundary $\partial \Omega_{F}$, yields the mechanical problem defined by:

$$
\left\{\begin{array}{l}
\nabla \cdot \boldsymbol{\sigma}(\mathbf{u}, d)+\mathbf{f}=\mathbf{0} \text { in } \Omega, \\
\mathbf{u}(\mathbf{x})=\overline{\mathbf{u}} \text { on } \partial \Omega_{u}, \\
\boldsymbol{\sigma} \mathbf{n}=\overline{\mathbf{F}} \text { on } \partial \Omega_{F} .
\end{array}\right.
$$

In (30), $\boldsymbol{\sigma}=\frac{\partial W}{\partial \varepsilon}$ is the second-order Cauchy stress tensor and $\overline{\mathbf{u}}$ are prescribed displacements on the boundary $\partial \Omega_{u}$. The constitutive law is expressed (see e.g. Nguyen et al. (2015)) by:

$$
\boldsymbol{\sigma}=\left((1-d)^{2}+k\right)\left\{\lambda\langle\operatorname{Tr} \boldsymbol{\varepsilon}\rangle_{+} \mathbf{1}+2 \mu \boldsymbol{\varepsilon}^{+}\right\}+\lambda\langle\operatorname{Tr} \boldsymbol{\varepsilon}\rangle_{-} \mathbf{1}+2 \mu \boldsymbol{\varepsilon}^{-} .
$$

Eqs. (28)-(30) are solved by a standard FE procedure in a staggered scheme at each time step (load increment). More theoretical and practical details can be found e.g. in Miehe et al. (2010); Nguyen et al. (2015).

It has been shown (see e.g Nguyen et al. (2016a); Amor et al. (2009); Kuhn et al. (2015); Benallal and Marigo (2007)) that $\ell$ is related to other material parameters and then should be identified experimentally as such. In section 4 , we conduct such identification by inverse analysis coupling simulation and experiments.

\subsection{Construction of Finite Element meshes from micro tomography images}

Finite Element meshes used for the numerical simulations are constructed from XR- $\mu \mathrm{CT}$ images of the samples in their initial stage, i.e. without micro cracks.

In the case of lightweight plaster (see Fig. 1), the geometry of the microstructure is quite simple, roughly constituted by a cylindrical domain containing spherical pores. Then, it is not necessary to convert each voxel into a single element, which would lead to prohibitive computational costs. Instead, we have used the microtomography image to determine the positions of the centres of the EPS beads (which are considered as pores) and their radii, and have constructed an idealized geometry involving the cylinder and spheres, which are meshed with tetrahedra. This simplification allows performing simulations 


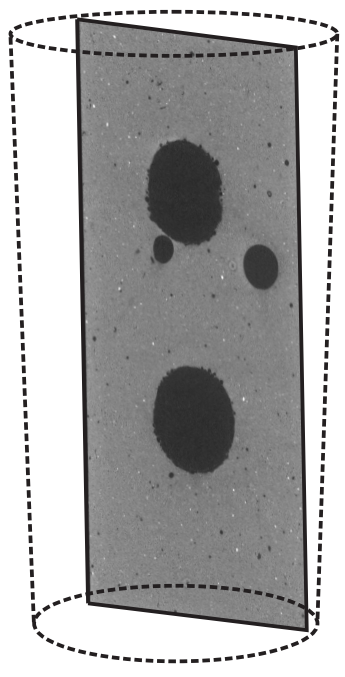

(a)

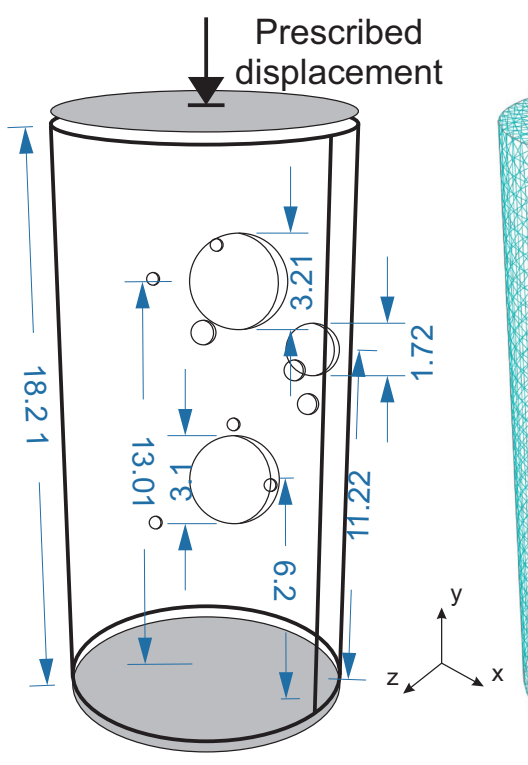

(b)

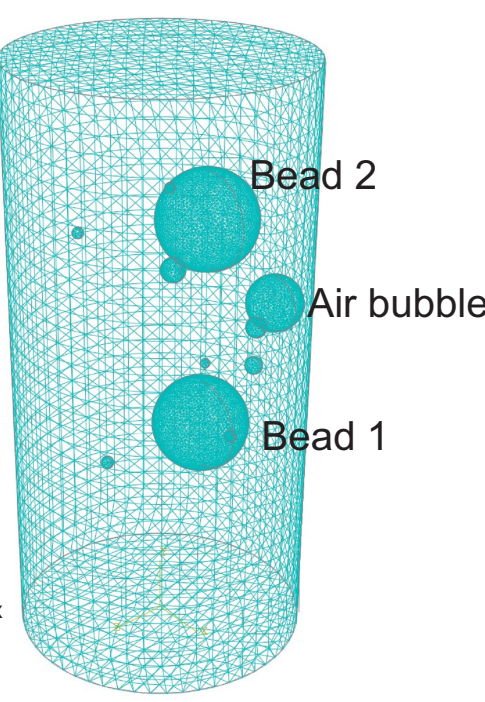

(c)

Fig. 5. (a) Microtomography image of the plaster sample PlasterS2 (vertical slice); (b) idealized geometry; (c) conforming mesh.

with much less elements than direct conversion of voxels to regular elements. Here, we neglect the smallest pores in the model (with diameters lower than 40 voxels). The positions and diameters of the pores are determined by using the plugin $3 D$ Objects Counter of the FiJi image processing software (Schindelin et al. (2012)). It is applied on the binary image resulting from a basic thresholding of the full CT volumes. Because of the clearly different grey levels of both phases and of the sharp interfaces, the choice of the grey level used to separate phases is not critical. An example of reconstructed idealized geometry for one sample of which a vertical cross-section of the XR- $\mu \mathrm{CT}$ image is shown in Fig. 5 (a), is provided in Fig. 5(b). The corresponding mesh is depicted in Fig. 5(c).

In the case of lightweight concrete (see Fig. 1(b)), it is very difficult to model the geometry with a collection of simple geometrical volumes (spheres, poyhedra...). Then, in the present work, we have converted each voxel to a cubic element and the properties of each phase have been mapped from voxels to the corresponding elements in a regular mesh. Segmentation of the voxels in the CT images has been performed by means of a simple thresholding based on the grey level of the CT-images, combined with some additional filtering routines. 


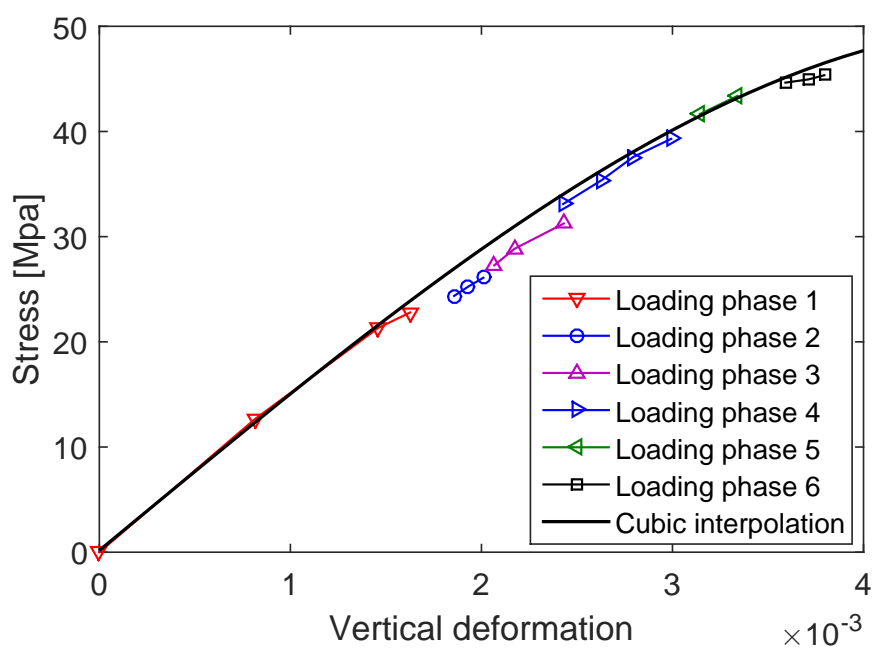

Fig. 6. Experimental stress - strain curve for the compression of the lightweight plaster sample PlasterS1 and cubic polynomial fit.

\section{Identification of material parameters $g_{c}$ and $\ell$ by inverse analysis}

The elastic parameters of the matrix phase (plaster) have been measured experimentally with uniaxial compression tests on samples manufactured with the same routes as samples PlasterS1 and PlasterS2, but containing no EPS beads (see more details in Nguyen (2015)). We have obtained $E=13.6 \mathrm{GPa}$ and $\nu=0.2$. To perform the numerical simulation of the lightweight plaster compression, the material parameters $g_{c}$ (toughness) and $\ell$ (process zone length) need to be specified. In this work, we have used an inverse approach based on a Newton-Raphson algorithm to identify these two material parameters from the experimental data of specimen PlasterS1 (see Fig. 6). For the sake of simplicity, the identification is restricted to a comparison between experimental and simulated global stress-strain curve. More elaborated procedures taking also into account local measurements available from the experiments, in the line of e.g. Heripre et al. (2007), are left for further investigations. Because the experimental curve is discontinuous (see Fig. 6), we replaced it by a cubic polynomial fit to obtain a continuous curve and to construct an error function.

The numerical finite element model used during each iteration of the inverse procedure involves 4 million elements. Monotonic compressive displacement increments of $U=-3 \times 10^{-4} \mathrm{~mm}$ are prescribed for 240 increments. We initialize the values of $g_{c}$ and $\ell$ from the experimental values identified for a similar material by Romani (2013) as $g_{c}^{0}=1.4 \mathrm{~N} / \mathrm{m}$ and $\ell^{0}=0.1 \mathrm{~mm}$. 
The cost function is here defined as:

$$
f\left(g_{c}^{k}, \ell^{k}\right)=\sum_{i}^{N}\left|\sigma_{i}^{\text {exp }}-\sigma_{i}^{\text {num }}\left(g_{c}^{k}, \ell^{k}\right)\right|
$$

where $N$ is the number of loading increments $(N=240)$ is the numerical overall stress obtained for given fracture toughness $g_{c}^{k}$ and process zone length $\ell^{k}$ at iterations $k$ at the $i^{\text {th }}$ increment of the numerical simulation and $\sigma_{i}^{e x p}$ is the stress associated to the corresponding computed overall strain according to the cubic fit of the experimental stress-strain curve. Note that at each iteration of the procedure, the whole loading of the sample is simulated to obtain the whole curve. Each iteration takes about $52 \mathrm{~h}$ on a 40 processors workstation running at $2.7-3.2 \mathrm{GHz}$ on the Matlab code developed at MSME Lab.

The procedure stops after 8 iterations, for a tolerance $f\left(g_{c}^{k}, \ell^{k}\right)=65.5 \mathrm{MPa}$, which corresponds to aa average distance of about $65.5 / 240 \approx 0.27 \mathrm{MPa}$ for each incremental step in the stress-strain curve. Recall that as the numerical derivatives and second-order derivatives of the residual are obtained by numerical perturbation, each iteration involves in total five 3D phase field computations (but independent) for the whole loading. The total procedure took $416 \mathrm{~h}$ to be achieved on 40 cores. For this error criterion, we obtained the values $g_{c} \approx 1.80 \mathrm{~N} / \mathrm{m}$ and $\ell \approx 0.0250 \mathrm{~mm}$. The evolution of the parameters with the iterations of the inverse analysis is depicted in Fig. 7. We note that our material compared with the one in the work of Romani (2013) is dryer (drying time is much longer), which induces that its strength is larger. This explains that the obtained $g_{c}(1.8 \mathrm{~N} / \mathrm{m})$ is larger than the one measured in the previous study of Romani et al. (2015) of about $1.4 \mathrm{~N} / \mathrm{m}$. The increased strength may also induce a larger critical stress $\sigma_{c}$, which is associated with a smaller process zone length $\ell$, (according to the analysis in Nguyen et al. (2016a)). Indeed $\ell$ was found to be $0.1 \mathrm{~mm}$ in Romani (2013) and is now identified to be four times smaller.

The same inverse procedure has been applied to sample PlasterS2, for which we obtained $g_{c}=1.76 \mathrm{~N} / \mathrm{m}$ and $\ell=0.0254 \mathrm{~mm}$. These values are very close to those obtained with sample PlasterS1, which provides some credit to our identification methodology. We have used $\ell=0.025 \mathrm{~mm}$ and $g_{c}=1.8 \mathrm{~N} / \mathrm{m}$ to perform the rest of the simulations on the plaster samples, the results of which are presented in section 6 .

In Nguyen et al. (2016a), a simple 1D analysis provided a rough relationships between $\ell, E$ and the tensile strength $\sigma_{c}$ as $\sigma_{c}=\frac{9}{16} \sqrt{\frac{E g_{c}}{3_{l}}}$. For the above identified values, we obtain $\sigma_{c} \approx 10 \mathrm{MPa}$. These parameters found by the present inverse analysis are of the same order of magnitude as compared to some available values from the literature: in Coquard and Boistelle (1996), Meille et al. (2003), the identified parameters for the plaster with 53-55\% 

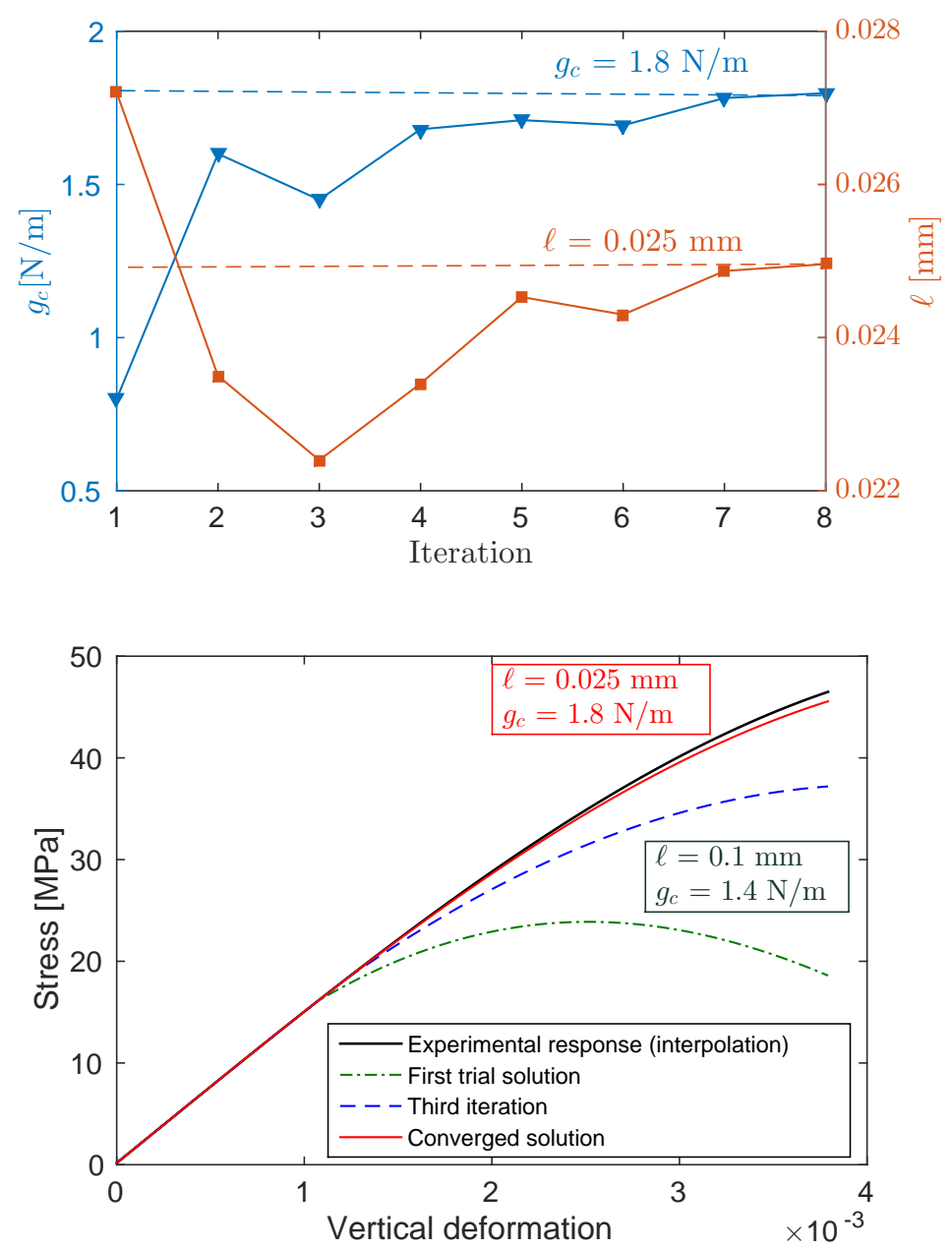

Fig. 7. (a) Evolution of the parameters $\ell$ and $g_{c}$ during the inverse identification; (b) evolution of the predicted response with the evolution of the identified material parameters and comparison with the fitted experimental curve.

porosity were found as $E=5.5 \mathrm{GPa}$ and $g_{c} \simeq 4.58 \mathrm{~N} / \mathrm{m}$. In Vekinis et al. (1993), the plaster with $62.5 \%$ porosity was associated with $E=4.5 \mathrm{GPa}$, $g_{c}=4.35 \mathrm{~N} / \mathrm{m}$, and $\sigma_{c}=3.2 \pm 0.6 \mathrm{MPa}$.

\section{Using experimental DVC measurements as boundary conditions on a sub-volume in numerical simulations}

As mentioned previously, meshing the whole sample from the microtomography image with small-scale microstructural details is not tractable for nowadays simulation capabilities. Therefore, we introduce here a technique of subvolume simulation: we model only a sub-volume encompassing some details of interest in the sample. Prescribing macroscopic uniaxial compression con- 
ditions on such a sub-volume would lead to a poor prediction of the local mechanical fields, because of the strong influence of boundary conditions on local fields within non representative volume elements of complex microstructures, and thus a potentially poor prediction of the local network of cracks. To circumvent this difficulty, we proposed to prescribe kinematic boundary conditions deduced from the experimental measurements of the local displacement by DVC performed on the XR- $\mu \mathrm{CT}$ images obtained during the in situ test. Such a methodology extends to three dimensions similar procedures already used in earlier studies on two dimensional models, aiming at comparing surface measurements of local fields within complex microstructures and corresponding predictions of numerical models (see e.g. Soppa et al. (2001); Heripre et al. (2007)).

\subsection{Systematic error correction}

To do so, a first difficulty to face is the noise and errors inherent to the experimental data obtained by DVC, which have to be filtered or corrected before these data can be used as Dirichlet boundary conditions in the finite element simulation. Indeed, these errors may induce non-physical gradients on the displacement field, and can yield spurious local stress concentrations near the boundaries of the sub-volume. Image correlation techniques, either in $2 \mathrm{D}$ or in $3 \mathrm{D}$, suffer from various sources of errors. Some are induced by image noise and are essentially random; they may only be reduced by low pass filtering or regularization techniques. A second source of errors, exhaustively discussed by Bornert et al. (2009), is due to the discrepancy between the physical transformation of the sample and the shape function used in the correlation algorithm to map reference and deformed images. In the present case of very small deformation gradients (below $10^{-3}$ ), this error can be neglected. A third one, known as the "systematic error" (Schreier et al. (2000)), is linked to the reconstruction of continuous grey levels from the discrete information provided by pixels or voxels, It consists in a systematic bias on the displacement components which is correlated with the fractional part of the actual value of the latter. An exhaustive discussion of its origins and dependences can be found in Amiot et al. (2013). Because of its systematic nature, it might be corrected if the so-called characteristic "S-shaped" curve which quantifies it can be determined for the specific experimental conditions of the test and the chosen image processing algorithm. A procedure based on the analysis of a pair of images of an undeformed sample taken at two slightly different magnifications has been proposed to do so by Dautriat et al. (2011) in the context of 2D imaging by SEM. It can be straightforwardly extended to 3D imaging by laboratory XR- $\mu \mathrm{CT}$ imaging, thanks to the continuous magnification provided by the cone-beam projection of a laboratory scanner. It has systematically been applied before the in situ compression tests, for the actual experimental conditions of the experiments, so that it makes sense to assume that the obtained 
S-shaped curve is also representative of the DVC systematic errors encountered when analysing the deformed images recorded during the compression test. An example of such systematic error curves for the three displacement components is provided by Figure 8 for a plaster sample. It is observed that, as expected, the error curves for the $\mathrm{X}$ and $\mathrm{Y}$ components are identical, with an amplitude below 0.1 voxels, while the error along the $\mathrm{Z}$ axis (i.e. parallel to rotation axis of the tomograph) is somewhat larger. This anisotropy of the error is a direct consequence of the reconstruction principle, for which $\mathrm{X}$ and $\mathrm{Y}$ axes are equivalent but $\mathrm{Z}$ is different.
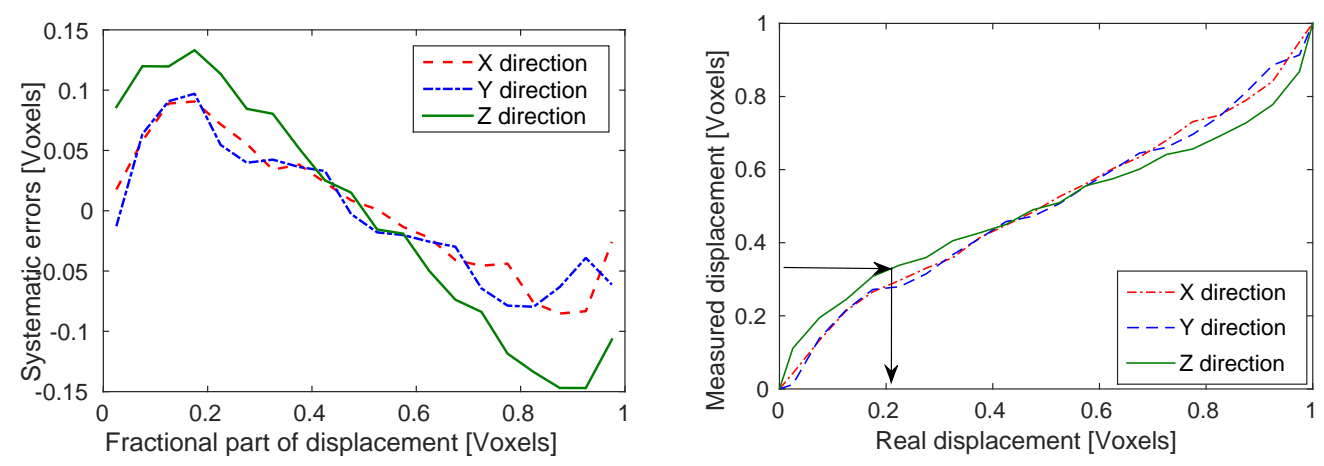

Fig. 8. (a) Systematic error analysis for the specimen PlasterS1; (b) relation between real and measured displacements.

Using the systematic errors curve, one can construct the bijective relations between measured and real fractional parts of the three components of displacement, as represented in Fig. 8(b). These relations have been fitted by polynomials of order 5 which have been used to remove the systematic bias from any measured fractional part of a displacement component, as suggested by the arrows in Fig. 8(b). Data correspond again to sample PlasterS1.

To illustrate the benefit of this procedure, we consider a sub-volume, where the displacements of a plane of investigation are corrected by using the above polynomial approximations. The $z$-displacements before and after correction are depicted in Fig. 9. We note that the systematic sub-voxels correction reduces the fluctuations of the displacement field. However the obtained values still exhibit some local spurious singularities, that may induce erroneous crack initiations. The detailed analysis of these problems will be considered in the next section.

\subsection{Definition of loading data}

After applying the above correction procedure for each loading step, the obtained displacement fields are used to construct the full displacement history to be prescribed at the boundary of the sub-volume. For this purpose, we have 


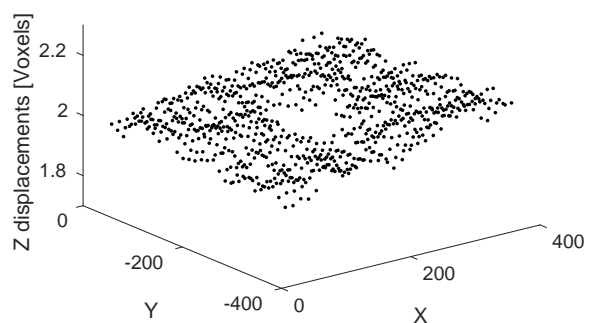

(a)

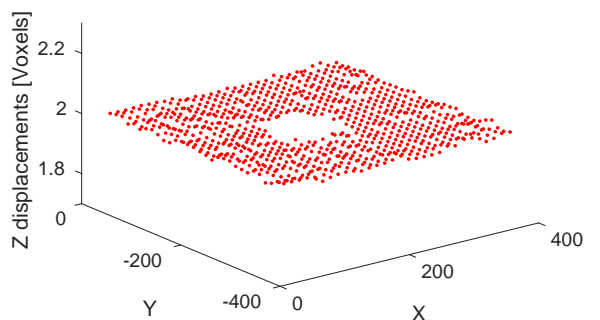

(b)

Fig. 9. $z$-displacement of a plane of investigation: (a) before correction; (b) after correction.

to face another issue if we consider an isolated point on the boundary: the displacement evolution is not smooth in time. To illustrate this phenomenon, we plot the load-displacement curve of one correlation point during the test in Fig. 10(a). This curve clearly shows a non-smooth evolution of the displacement. These apparent fluctuations may have various sources. First recall that the sample has undergone several loading-unloading stages, because the test lasted several days and the sample has been partially unloaded during nights. These loading cycles may induce overall rigid body motions of the sample. More generally, the overall behaviour of the compression device might also induce such motions at sample scale, because it is not perfectly rigid. A procedure will be introduced in the future to subtract these rigid body motions. Another possible source of fluctuations are the above mentioned DVC random errors. They can also be quantified with the procedure used to evaluate systematic errors (Dautriat et al. (2011)), and are of the order of 0.1 voxel, which is close to the order of magnitude of some of the observed fluctuations in Fig. 10(a). Some smoothing is then required to filter the DVC data.

For the sake of simplicity, and to remove all possible sources of spurious local displacement fluctuations which could induce non-physical damage, we propose to filter the DVC measurements at each point of the boundary of the sub-volume, by a quadratic fit with respect to time, as illustrated in Fig. 10(b). This quadratic fit will also serve as a temporal interpolation to define displacements at numerical loading increments which do not coincide with experimental load steps.

Finally, the correlation points are usually not the same as the nodes used for boundary conditions. Therefore, an interpolation is needed to compute displacements for each node at the boundaries of the sub-volume. We use here the same principles as those used to construct the subtracted image: we define the approximate transformation $\boldsymbol{\Phi}_{N}$ of $\boldsymbol{\Phi}$ near a FEM node as the first order fit of the displacement of $N$ neighbour positions successfully investigated by DVC. This procedure is illustrated in Fig. 11 and more details can be found in Nguyen (2015). 

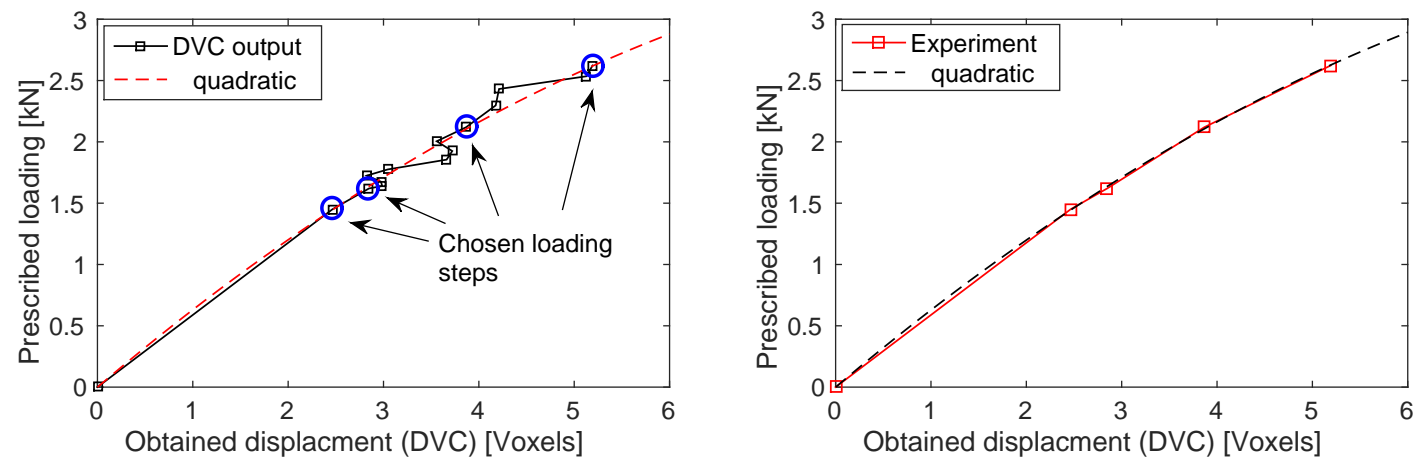

Fig. 10. Displacement of a particular voxel with respect to time obtained by DVC during the in situ test and its quadratic polynomial fit. Data obtained for PlasterS2 sample.

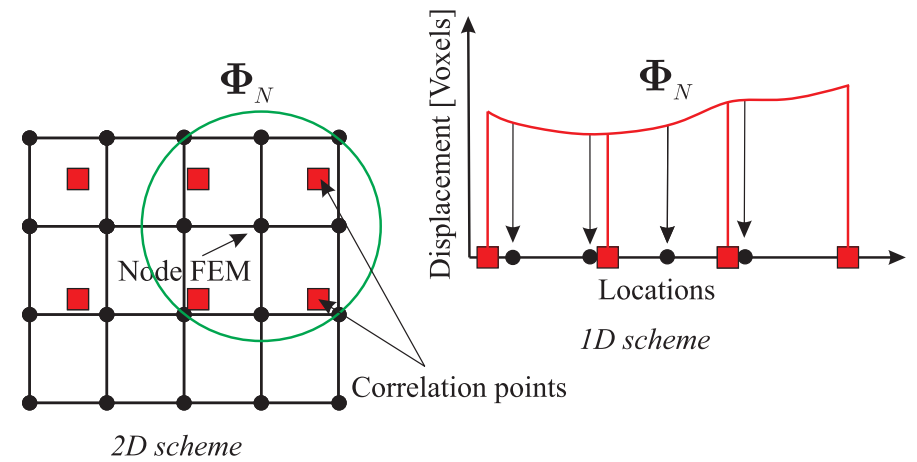

Fig. 11. Extraction of FE boundary conditions from DVC data: interpolation procedure.

\section{Direct comparisons of microcracking}

We now conduct direct comparisons between 3D crack networks observed experimentally during the in situ compression tests and numerical predictions in both lightweight plaster and concrete samples at the scale of the heterogeneities. We emphasize the following remarks:

- The damage mechanical parameters for lightweight plaster samples have been identified by the inverse numerical-experimental procedure described in the section 4 .

- The numerical model does not contain initial cracks.

- The initial numerical models describe the exact same microstructural geometries as the experimental tests, except for details smaller than some typical size specified later, which are neglected.

- Cracks are initiated and propagated in the numerical simulations without any a priori assumption about their path, and no update of any parameter is made during the simulation with respect to the experimental data.

- The comparisons are made between the numerically predicted cracks and 


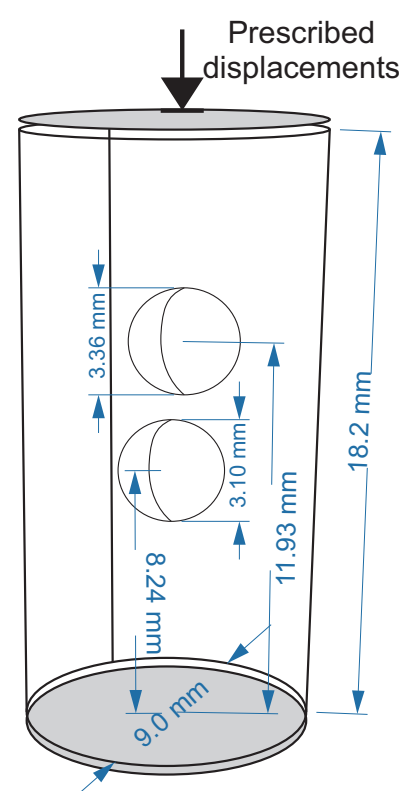

(a)

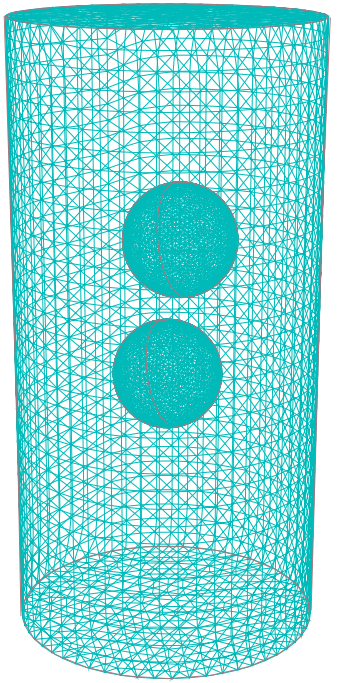

(b)

Fig. 12. Idealized model used as input of numerical simulation of PlasterS1 sample: (a) geometry and boundary conditions; (b) detailed view of the mesh.

the experimental ones detected by image processing for the same loads.

\subsection{Global comparisons: lightweight plaster}

\subsubsection{PlasterS1}

We focus here on the lightweight sample plasterS1. In this test, we only take into account the EPS beads, and neglect the micro porosity and small pores. The obtained geometry for the considered test is depicted in Fig. 12. Recalling that the identified process zone length is $\ell=0.0254 \mathrm{~mm}$, we have used unstructured refined meshes such that the characteristic size of the elements in the zones where the cracks propagate is $\mathrm{h}_{\min }=0.0125 \mathrm{~mm}$. In zones away from the cracks, the typical size of the elements is $h_{\max }=0.35 \mathrm{~mm}$. The whole mesh contains about 12 million elements.

During the experimental procedure, two polished PMMA plates were used to remove spurious crack initiation from the top and bottom faces of the sample, which were observed in preliminary tests. In the simulation, uni-axial compression is prescribed (see Fig. 12): on the lower surface, the $z$-displacements are blocked, while on the upper surface, the $z$-displacements are prescribed. The $x$ and $y$-displacements are free on these two surfaces.

In the simulation, monotonic compressive displacement increments $\bar{U}=-5 \times$ 


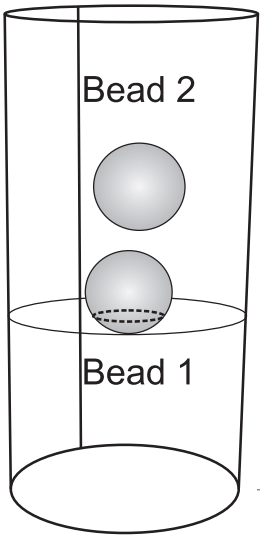

(a)

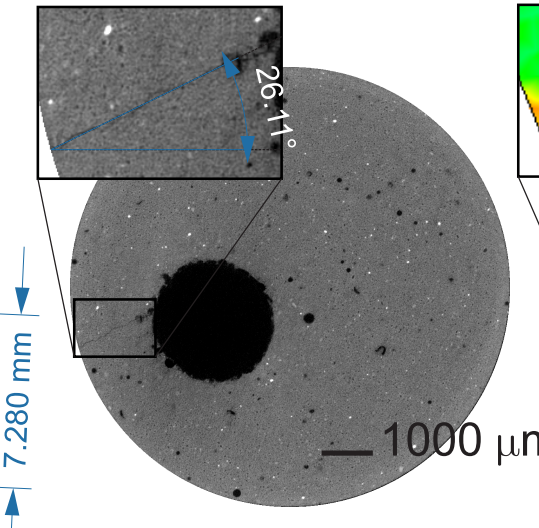

(b)

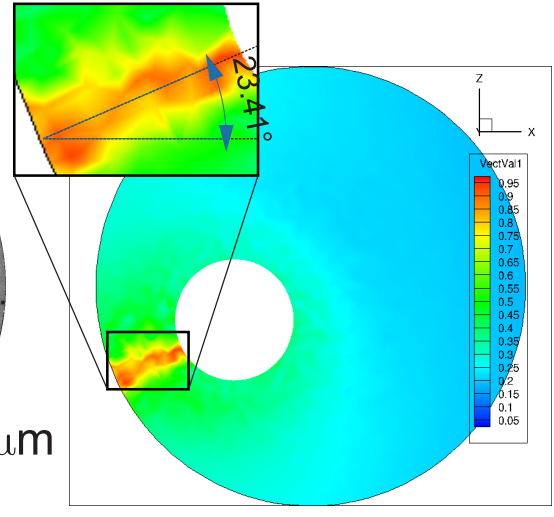

(c)

Fig. 13. Crack path comparison in a region bellow Bead 1: (a) position; (b) experimental result; (c) simulation result.

$10^{-4} \mathrm{~mm}$ have been prescribed as long as the value of $d$ was lower than 0.9 at any integration point, and then decreased to $\bar{U}=-5 \times 10^{-5} \mathrm{~mm}$.

The transformed image was used here for direct comparison with numerical simulation. The three-dimensional crack paths observed in the experiment and predicted by the computation are compared at the last step of the loading $(\mathrm{F}=2.885 \mathrm{kN})$, in two regions near the two EPS beads, called "Bead 1" and "Bead 2" in Fig. 13(a).

First, we compare the trace of the 3D crack network in a plane located at the height $H=7.28 \mathrm{~mm}$ from the bottom of the specimen, and cutting the Bead 1. We observe, in the experiment, a crack linking the outer surface of the sample and the border of the lower bead, as shown in Fig. 13(b). The same crack morphology is clearly observed in the numerical simulation, as shown in Fig. 13(c). We remind here that in the numerical simulation, we start from an un-notched specimen and did not make any assumption about where the cracks initiate and propagate. To make a quantitative comparison, we have analysed the angle between the crack and the $x$-axis of the image in the plane of observation defined in Fig. 13(b) and (c) for both the experiment and the numerical simulation. The obtained values are about $26.1^{\circ}$ for the experiment, and $23^{\circ}$ for the numerical simulation, showing a very good agreement. However, the location of crack initiation for both observation is slightly different: in the numerical simulation the crack follows the closest path from bead to boundary of the sample. In the experiment, it is slightly shifted. The reason for that is probably that the real crack path was deviated by the presence of some micropores, which can be seen in Fig. 13(b), and which are not taken into account in the simplified ideal geometry of the numerical model.

Secondly, we compare the trace of the 3D crack network in a plane at the height $H=9.73 \mathrm{~mm}$ from the bottom of the specimen, and located between the two 


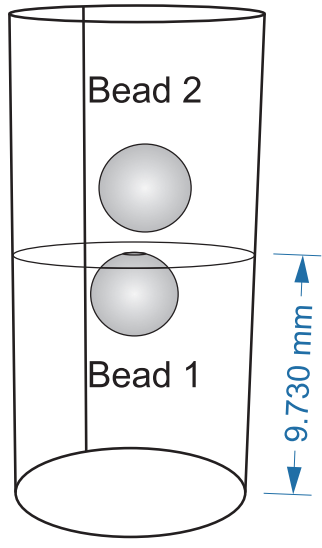

(a)

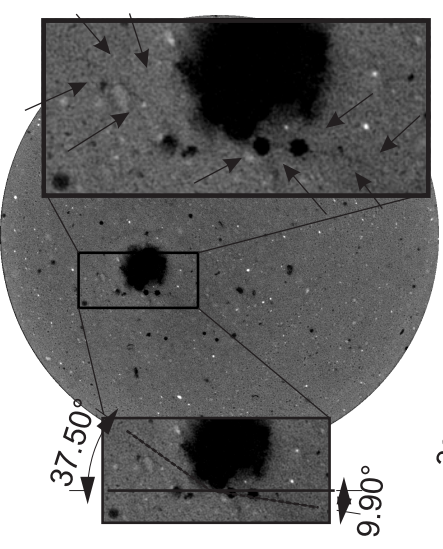

(b)

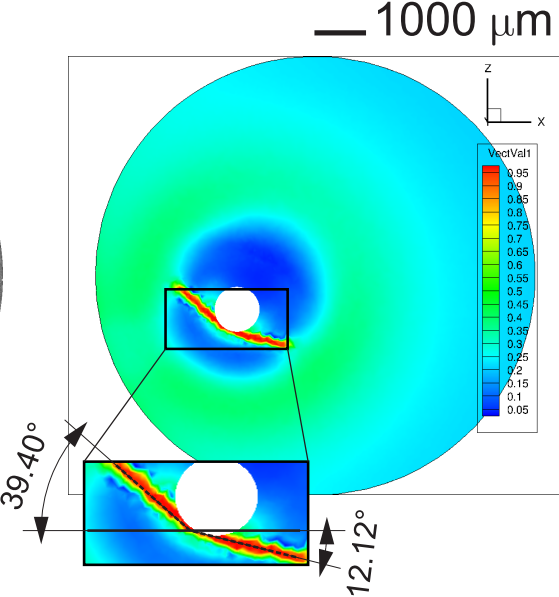

(c)

Fig. 14. Crack path comparison in a region above Bead 1: (a) position; (b) experimental result; (c) simulation result.

beads in Fig. 14(a). The experimental crack path is compared to the simulated one in Figs. 14(b) and 14(c), showing that the numerical simulation is again able to capture the position and morphology of the crack in a satisfactory way in this complex configuration. Again the orientations of both visible branches of the crack are reproduced with an accuracy of a few degrees. The remaining small discrepancies might again be linked to small morphological features of the pore which are note taken into account in the simulation.

A third comparison is performed in a plane located at the height $H=10.53 \mathrm{~mm}$ in Fig. 15(a), which crosses the second bead. Here again, the morphology of the crack network is correctly captured. Several geometrical features are quantitatively compared in Figs. 15(b) and 15(c) and a rather good agreement is observed, in terms of both length and orientation of the cracks. We recall that comparisons are performed for the same macroscopic load level of $2.88 \mathrm{kN}$.

The last plane of investigation is located at the height $H=13.70 \mathrm{~mm}$ (see Fig. 16(a)), tangent to the top of Bead 2. This zone is interesting because in compression, we observe three crack surfaces roughly developing with an angle of $120^{\circ}$ from a point located on top of the spherical void (bead), as shown in Fig. 16(b). Remarkably, the simulation captures the same morphology of the crack network, both in terms of the relative orientation between the three connected cracks and the overall orientation of the crack network with respect to the microgeometry of the sample (see Fig. 16(c)).

These results, showing a direct comparison between the formation of a 3D complex crack network in an experiment and in a numerical simulation, are to our knowledge the first of their kind and prove the high level of predictive ability of the phase field method to simulate complex crack propagation in complex heterogeneous materials. We emphasize also the fact that these 


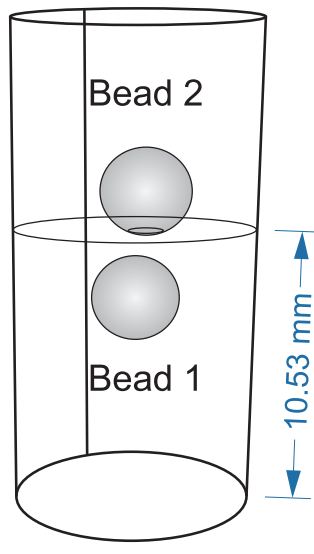

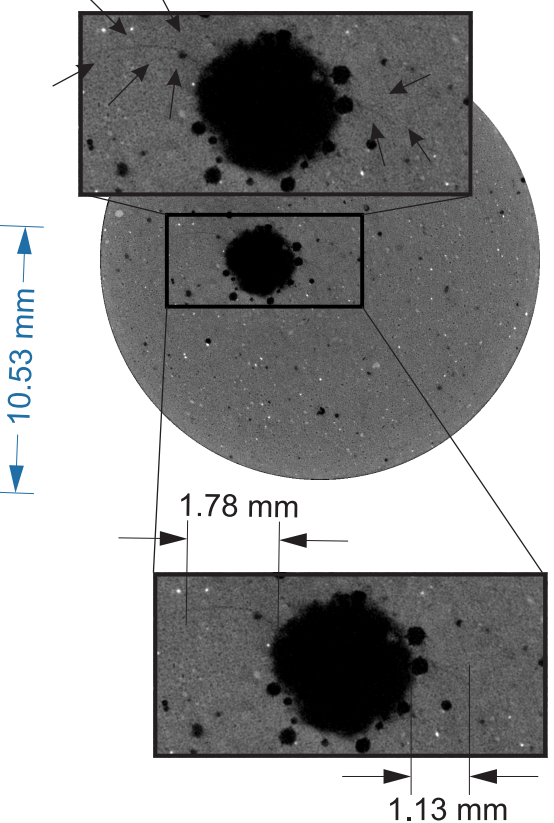

(b)

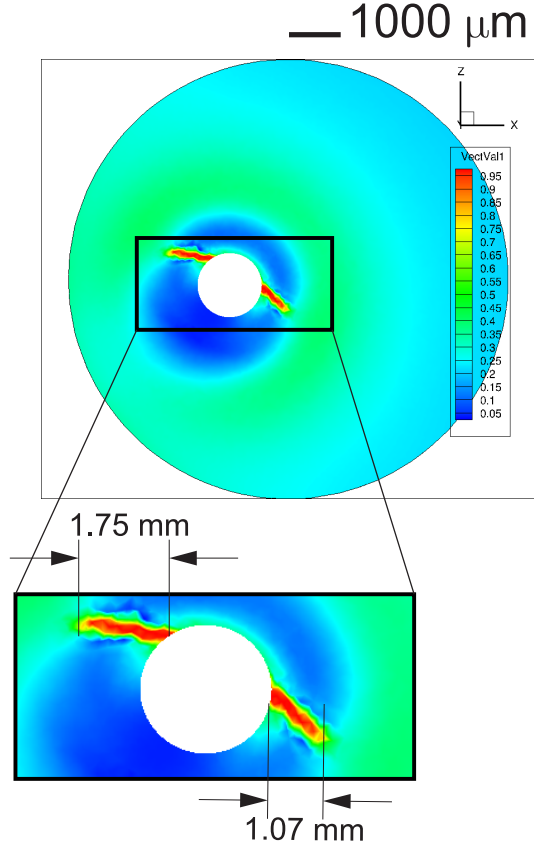

(c)

Fig. 15. Crack path comparison in region below Bead 2: (a) position; (b) experimental result; (c) simulation result.

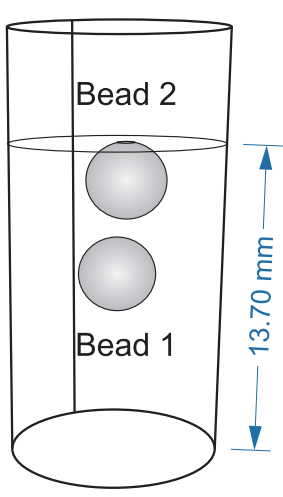

(a)

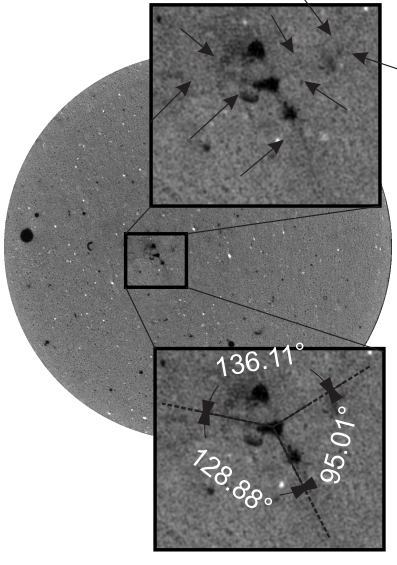

(b)

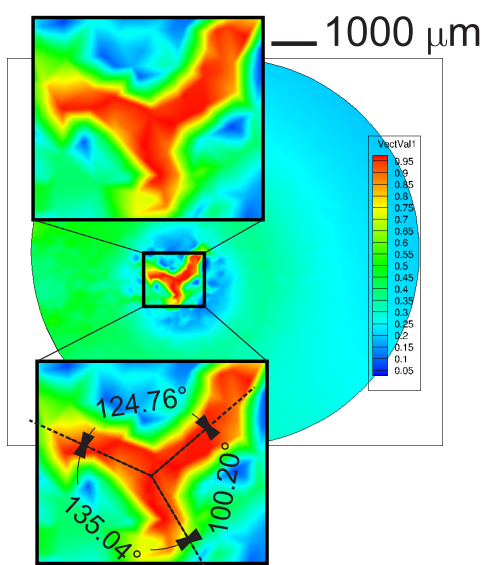

(c)

Fig. 16. Crack path comparison in a plane just above Bead 2: (a) position; (b) experimental result; (c) simulation result.

computations rely on simple isotropic elastic and fracture models, depending on a very limited number of parameters (two scalar parameters for isotropic elasticity and two others for damage).

In addition, these results evidence also that crack propagation, even in brittle materials for which some unpredictable randomness is often assumed, is essentially deterministic and can be assessed as soon as the main sources of the cracking phenomenon are taken into account in the numerical model. In the 
present case, the main factor that determines the location of cracking seems to be the heterogeneity of the elastic stress and strain field induced by the particular geometry of the large beads within the sample. The additional randomness induced by smaller pores seems to have only a second order influence. This will be confirmed in the next section.

\subsubsection{PlasterS2}

We present now comparisons of 3D crack propagation between experiments and simulations in the sample PlasterS2 containing multiple pores (see details in section 2). These pores consists in two EPS beads, a large bubble of air, and several smaller bubbles of air, as depicted in Fig. 5 .

As presented in Fig. 4, the first crack has been detected at $F=1.62 \mathrm{kN}$, while for the previous sample (PlasterS1), the first cracks initiated at $F=2.76 \mathrm{kN}$. This might be explained by the larger porosity of the second specimen. Furthermore, the large pores and beads are located near the boundary, inducing weaker zones. Here, small pores with diameters larger than 40 voxels (i.e. 320 $\mu \mathrm{m})$ are kept in the numerical model of the whole sample. An unstructured and adaptive mesh of 11 million tetrahedra has been constructed, with minimum and maximum element sizes $\mathrm{h}_{\min }=0.0125 \mathrm{~mm}$ and $\mathrm{h}_{\max }=0.35 \mathrm{~mm}$. The detailed geometry, mesh and boundary conditions are depicted in Fig. 5. The material parameters are the same as in the previous example (PlasterS1). Monotonic compressive loading steps $\bar{U}=-3 \times 10^{-4} \mathrm{~mm}$ are prescribed as long as $d_{\max }<0.9$ and then set to $\bar{U}=-3 \times 10^{-5} \mathrm{~mm}$.

The subtracted and transformed experimental images have been used for comparisons with the predictions of the simulations performed with the phase field method on the initial model described above.

First, a plane parallel to the section of the sample located above Bead 2 has been chosen, as depicted in Fig. 17(a). The plane cuts the EPS bead together with two small pores. The evolution of the 3D crack network is compared between experiment and simulation for steps 3, 6 and 10 in Figs. 17(b),(c) and $(\mathrm{d})$.

Experiment and simulation results agree qualitatively well, at least in terms of location and orientation of the cracks. Nevertheless, we observe here a significant difference in the crack length, that will be explained below by considering a longitudinal plane of observation..

Indeed, the crack network is now studied in a vertical plane, again for loading steps 3, 6 and 10. Results of the simulation are provided in Fig. 18 (a), (b) and (c). We can observe mainly two vertical cracks initiating from Bead 2. Experimental results are provided in Figs. 18 (d), (e), (f), showing a similar 


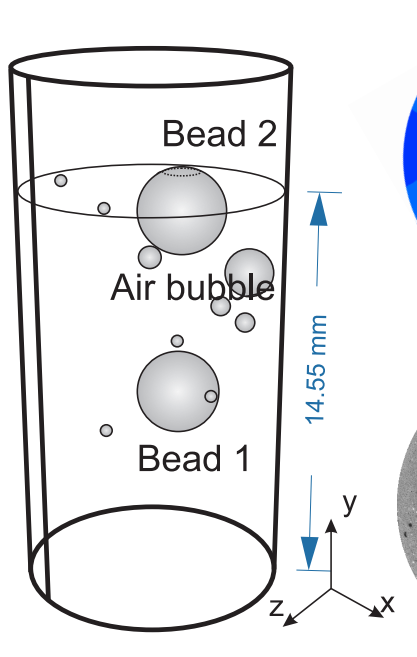

(a)
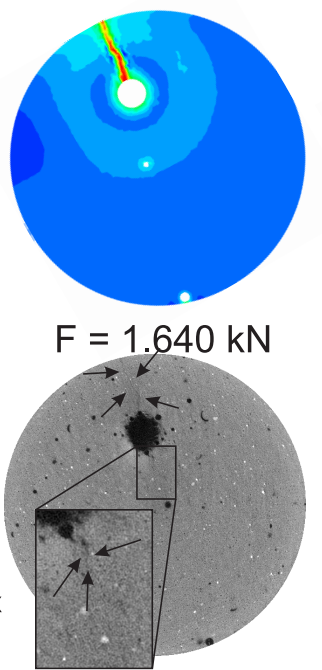

(b)
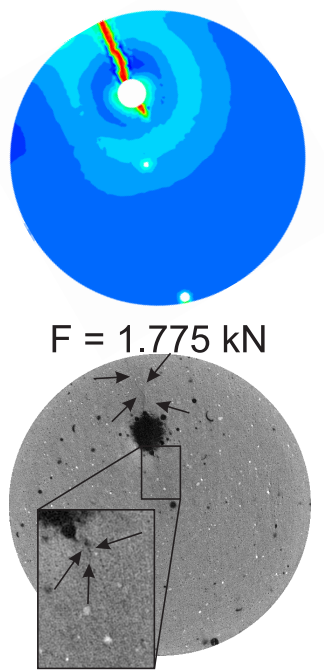

(c)
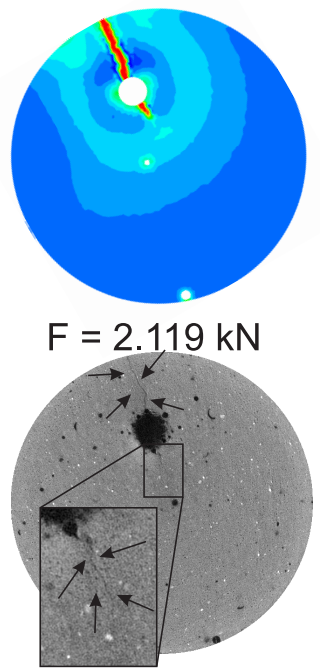

(d)

Fig. 17. Crack path evolution in a region above Bead 2: (a) Position of the plane chosen for the comparisons; upper images (in color): numerical simulations; lower images: experiments (transformed images); (b) loading step 3; (c) loading step 6; (d) loading step 10.

cracking pattern and a good agreement regarding crack length for the lower crack. However, the length of the crack heading toward the upper end is here not validated: its propagation is shorter in the simulation than in the experiment. A possible explanation might be due to the ideal Dirichlet boundary conditions in the simulation which might not reflect the actual ones in the experiment. Indeed, vertical displacements are prescribed uniformly on the upper and lower ends of the sample. A crack propagating towards these ends will thus not be allowed to develop a displacement discontinuity with a vertical component when it gets close to the ends. Conversely, the propagation towards the ends of a crack presenting such a discontinuity will be difficult. To overcome this limitation of the numerical simulation, one should use less strict displacement boundary conditions. A solution might be to include in the model the softer PMMA plates used in this test, together with unilateral jump conditions at the plate/sample interface. Such more complex computations are left for further investigations.

The rupture of the sample occurs on the top half part. The cracks nucleate and propagate mainly in a region near Bead 2 and the biggest bubble of air. Views of the simulated and of the experimental cracks are provided in Fig. 19. Again a very nice agreement between the model and the experiment is observed.

For the last comparison, we chose a horizontal plane passing below Bead 2 and above an air bubble, at height $H=11.9 \mathrm{~mm}$ (see Fig. 20 (a)). Comparisons between experimental and numerical results are depicted in Figs. 20(b)-(c). 


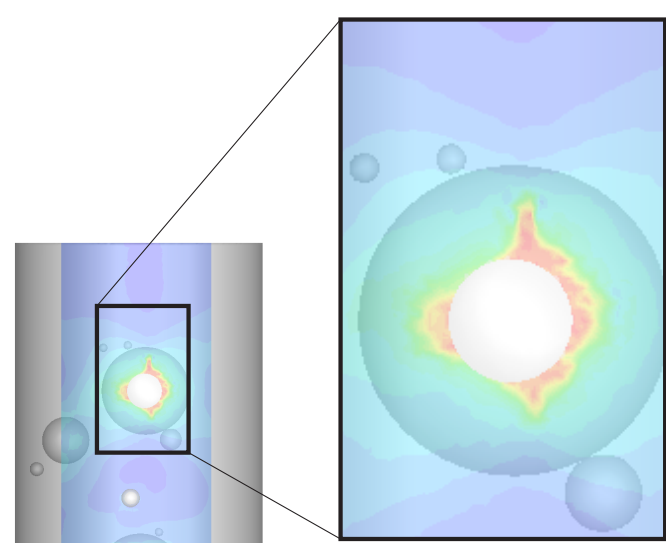

(a)

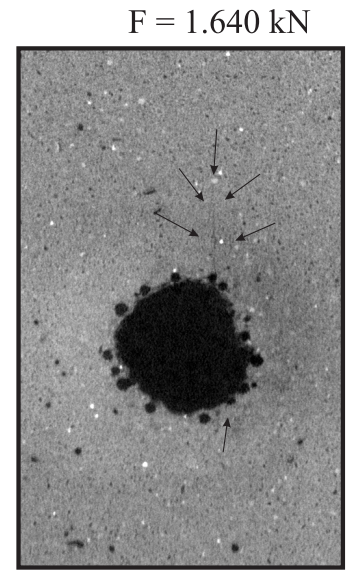

(d)

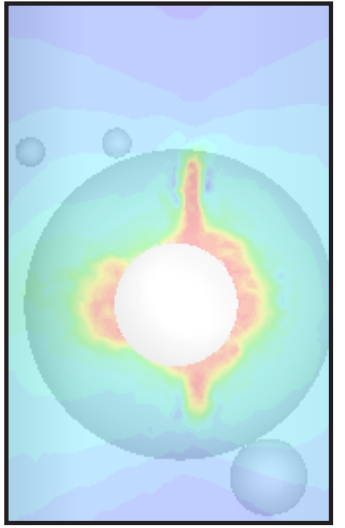

(b)

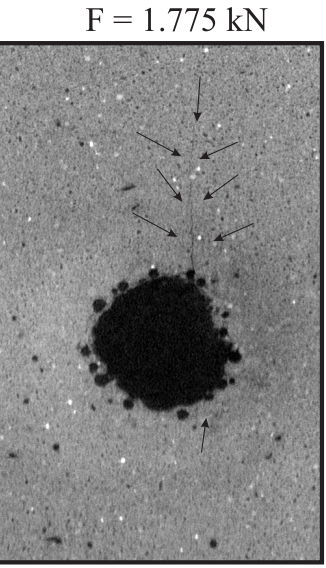

(e)

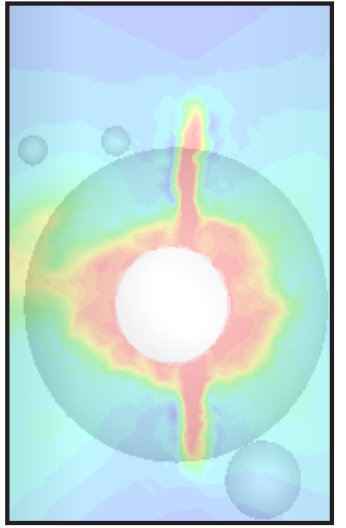

(c)

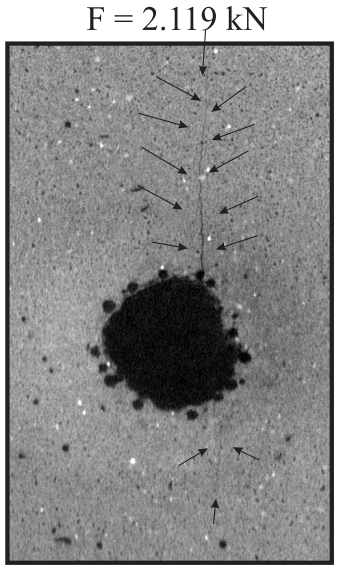

(f)

Fig. 18. Comparisons in a vertical plane. Upper images (in color): numerical simulation; lower images (grey levels): experiments. (a), (d); loading step 3; (b), (e): loading step 6; (c), (f): loading step 10.
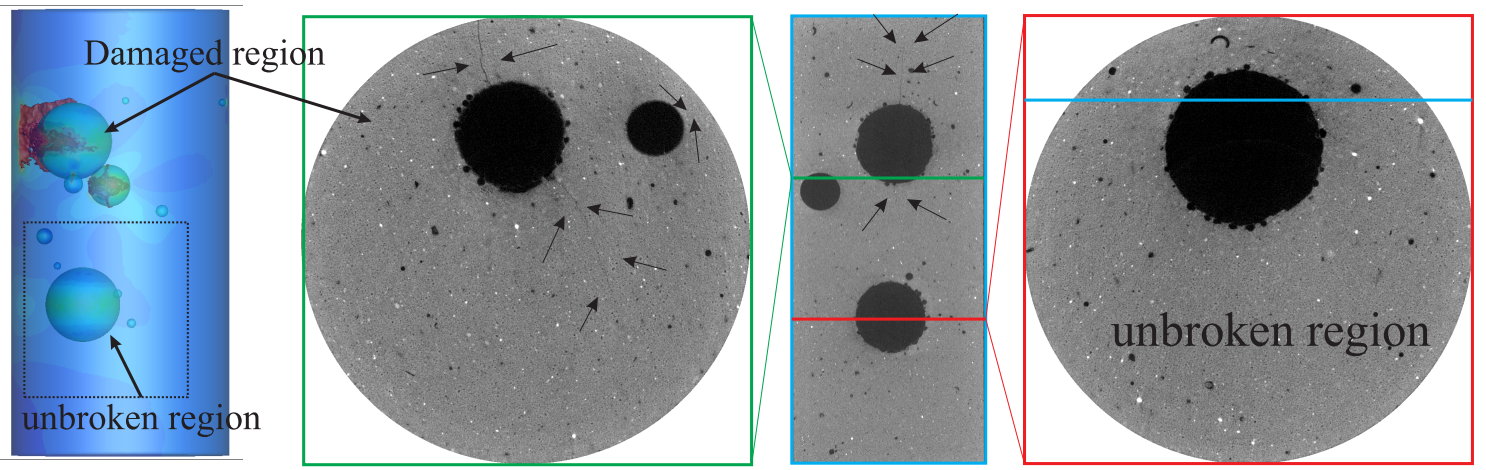

Fig. 19. View of the damage zone in the lightweight plaster sample PlasterS2 $(F=2.53 \mathrm{kN})$.

Finally, we show in Fig. 21 (a) the experimental image of the fully developed failure crack network within the PlasterS2 sample just after the load $F=$ $2.71 \mathrm{kN}$. The simulation shown in Fig. 21 (b) is plotted for $F=2.64 \mathrm{kN}$, which does not correspond to the same load. We could not carry out the 


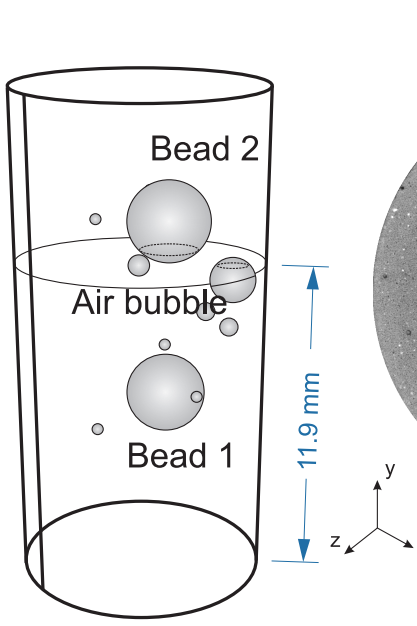

(a)

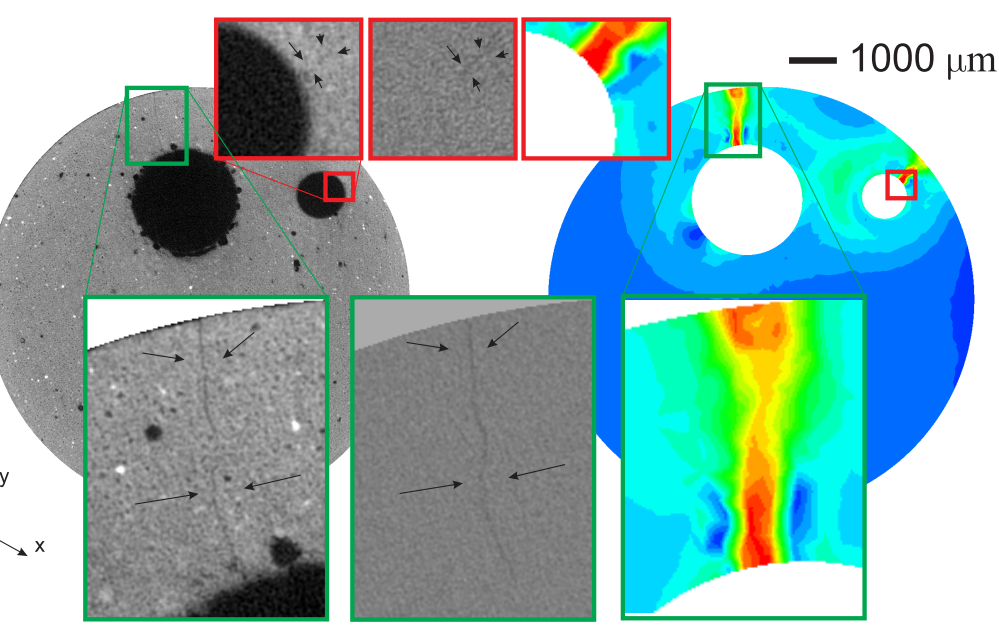

(b)

(c)

Fig. 20. Crack path in a region below Bead 2: (a) position of the observation plane; (b) Experimental cracks (transformed et subtracted images); (c) Simulated cracks $(F=2.12 \mathrm{kN})$

simulation up to the fully broken state of the sample because the required fine mesh along all crack paths would lead to computational times above our present capabilities. In addition, the modelling of the boundary conditions, here idealized as Dirichlet displacement boundary conditions, tends to block the cracks before they come out onto the top and bottom surfaces of the sample where the load is applied. However, we can note that the main cracks and their actual topologies are captured in the simulation in their early propagation stage.

\subsection{Sub-volume comparisons}

As commented above, numerical simulations compared well to experimental observations in terms of overall features of the crack network spreading throughout the whole sample, at the scale of the larger microstructural heterogeneities of the sample. Some more local features could not be reproduced, possibly because of their dependence with details of the real microstructure not taken into account by the model. The purpose of this section is to check whether a better prediction of these local features can be obtained with more precise numerical models. To do so, and in order to limit the numerical cost of the simulations, sub-volumes of the sample will be considered now, with a refined description of their geometry. The specific procedures described in section 4 to prescribe meaningful experimental displacements to the boundaries of these sub-volumes have been used for the calculations presented here. This has been performed first on the PlasterS1 sample and then to the lightweight concrete with the more complex microstructure. 


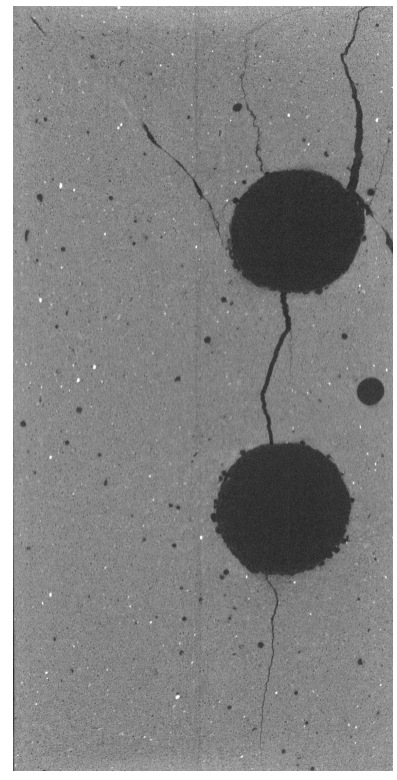

(a)

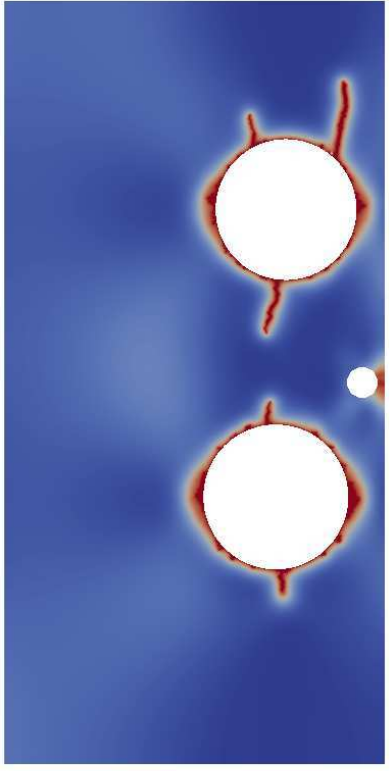

(b)

Fig. 21. (a) Experimental crack network at fully broken state of PlasterS2 sample just after the load $F=2.71 \mathrm{kN}$; (b) simulated crack at an early stage of propagation $(F=2.64 \mathrm{kN})$.

\subsubsection{Lightweight plaster (PlasterS1)}

We focus first on PlasterS1 sample, and develop numerical models able to capture the effects of pores much smaller than the ones used in the previous models describing the whole sample.

A sub-volume of dimensions $350 \times 350 \times 30 \operatorname{voxel}^{3}\left(\right.$ or $\left.2.8 \times 2.8 \times 0.24 \mathrm{~mm}^{3}\right)$ is taken below Bead 2 (see Fig. 22). To ensure the detection of very tiny crack, we keep the resolution of the initial image for the input geometry for the simulation (i.e. a voxel is transferred into an 8-node element). All elements in pores are removed to reduce the computational costs.

Concerning the definition of boundary conditions, a first attempt has consisted in a direct application of the experimental displacements obtained from DVC, and extended to all nodes of the boundary of the sub-volume by the same interpolation procedure as that used to construct transformed and subtracted images (see section 3 and end of section 4). This however generated unphysical damage near the boundary as a consequence of the measurement errors of the unfiltered DVC data, as illustrated in Fig. 23. A second attempt has consisted in blindly running a median filter over the DVC data at each loading step to remove spurious singularities. Boundary effects could be removed but the obtained results were still not satisfactory, showing completely different crack paths between simulation and experiment. This issue might come from the filtering process, which excessively smoothes out the displacement discontinu- 


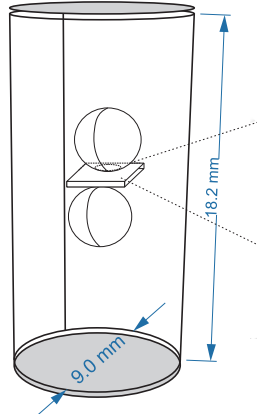

(a)

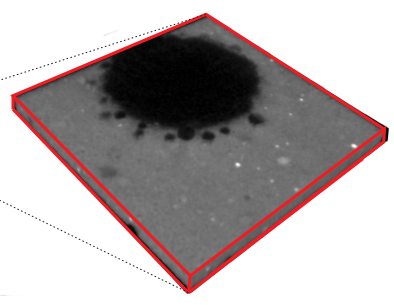

(b)

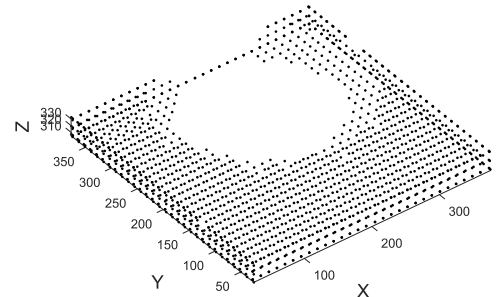

(c)

Fig. 22. Sub-volume considered for comparisons of micro cracking propagation in the lightweight plaster sample PlasterS1: (a) location in the sample; (b) associated $\mathrm{CT}$ image; (c) 3D correlation points on the surface used to define the boundary conditions.

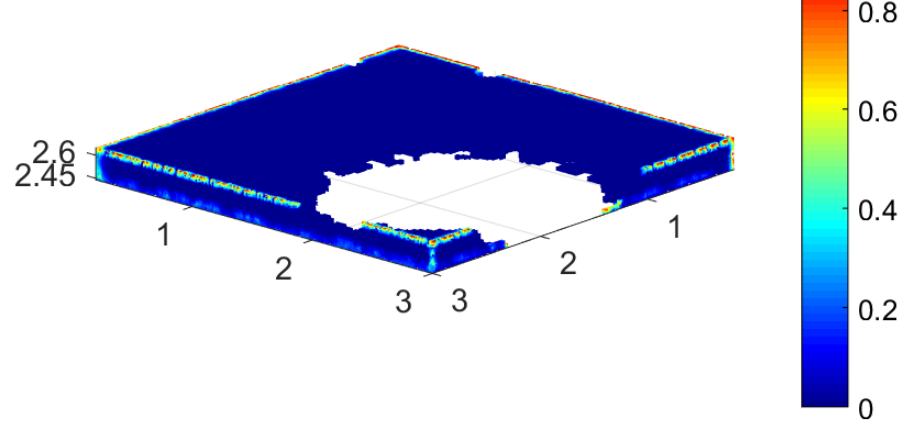

Fig. 23. Spurious damage on the boundary of the domain used for the simulation due to noise in the DVC data obtained from the experiments.

ities on the boundary of the sub-volume, that do actually exist when the crack crosses the boundary of the sample in the experiment. In addition, systematic errors (as discussed in section 4) might induce unphysical discontinuities which may generate unphysical cracks. To overcome these difficulties, the whole set of procedures described in section 4 have been developed and applied to the considered sub-volume. The location of the 3D correlation points on the surface of the sub-volume used to define the boundary conditions in shown in Fig. 22 (c).

We compare the evolution of micro cracks in the sub-volume for the three loading steps 15, 17 and 19. Results for step 15 (load $F=2.65 \mathrm{kN}$ ) are depicted in Fig. 25. The black and red colours refer to existing pores and cracks, respectively. We note that the crack on the right side of the EPS bead is well captured for both experiment and simulation, but the crack on the left is not observed in the experiment. This might be due to the image treatment 


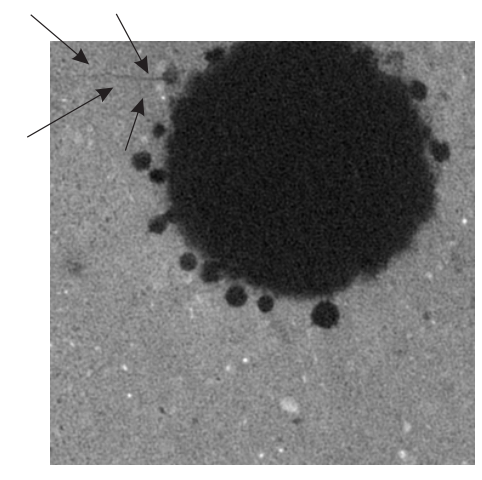

(a)

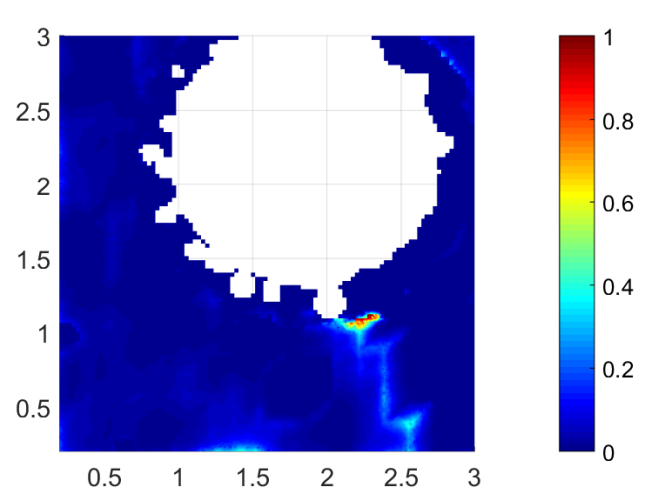

(b)

Fig. 24. Differences in crack morphology observed when smooth filtered DVC data are prescribed to the boundary of the sub-volume: (a) cracks detected from experiment; (b) crack obtained by numerical simulation.

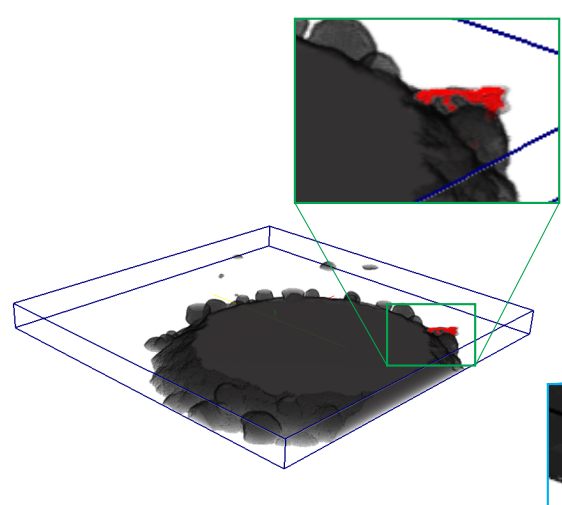

(a)

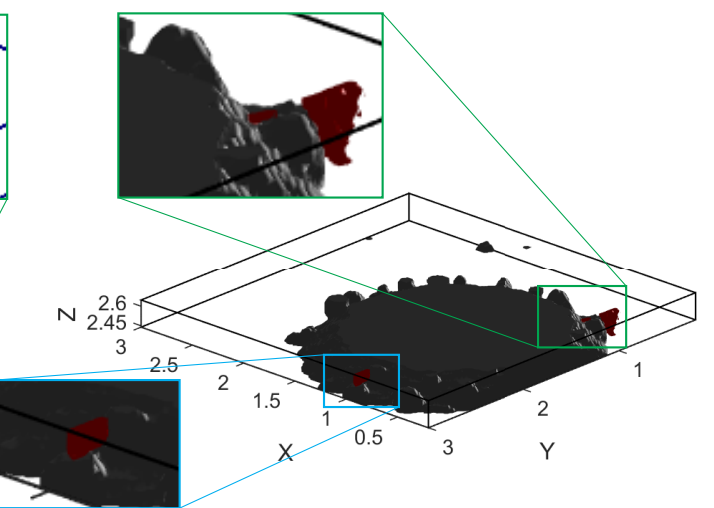

(b)

Fig. 25. 3D crack path in the sub-volume of lightweight plaster (PlasterS1) for loading step 15: (a) crack extracted from experiment; (b) numerical simulation.

which does not allow detecting very short cracks in the early stage of initiation.

However, confirming this assumption, for steps 17 and 19, we can detect the left crack also in the experiment, as seen in Figs. 26 and 27, so that a good agreement is indeed obtained between the experiment and simulation run with such boundary conditions.

In order to perform quantitative comparisons, we have chosen a plane (see Fig. 28) passing through the sub-volume and have compared the length of one crack for several loadings. Results are compared in Fig. 29. Remarkably, we observe similar crack lengths for all three loading steps. One should again keep in mind that in the simulation there is no a priori assumption about the initiation location and path of the crack and that material parameters (except elastic parameters) have been identified by an inverse approach. Finally, the simulation and experiments are compared for the same load. 


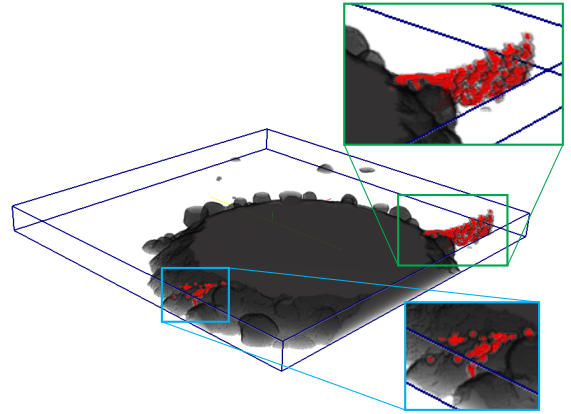

(a)

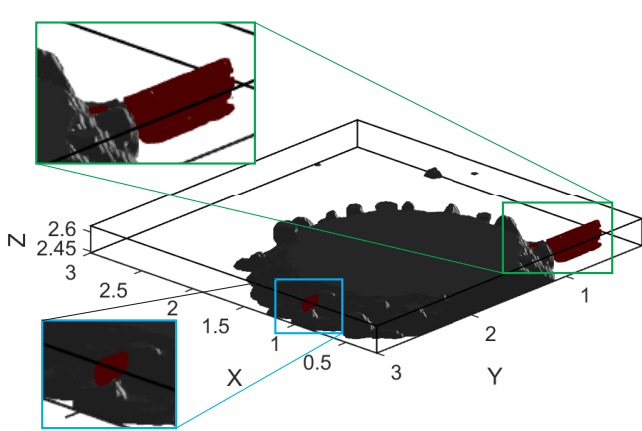

(b)

Fig. 26. 3D crack path in the sub-volume of lightweight plaster for loading step 17: (a) crack extracted from experiment; (b) numerical simulation.

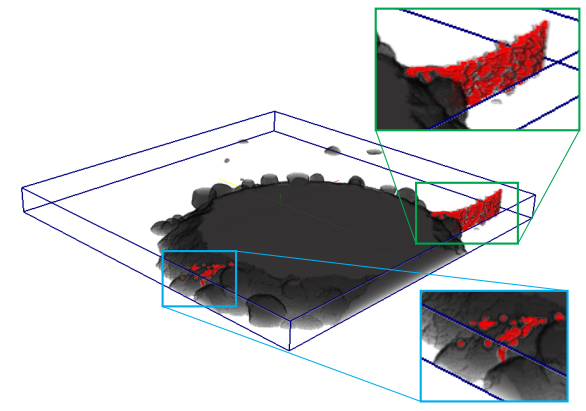

(a)

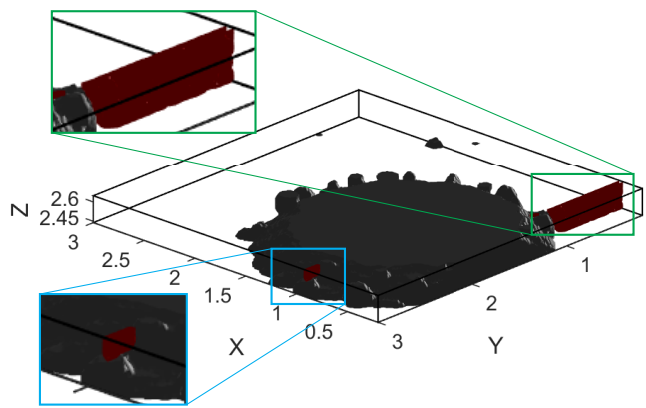

(b)

Fig. 27. 3D crack path in the sub-volume of lightweight plaster for loading step 19: (a) crack extracted from experiment; (b) numerical simulation.

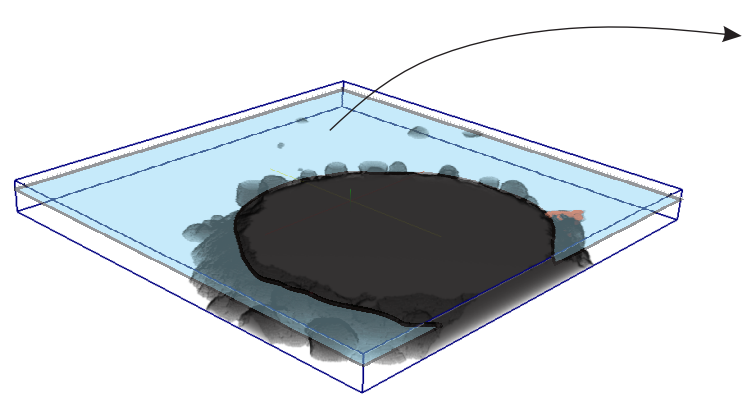

(a)

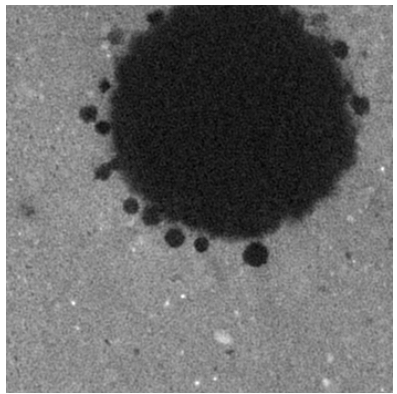

(b)

Fig. 28. Definition of plane of investigation: (a) location in sub-volume; (b) XR- $\mu \mathrm{CT}$ images of loading step 17.

\subsubsection{Lightweight concrete}

In this section, we compare the micro cracking network propagation obtained from the experiments and the numerical simulations in the case of the lightweight concrete sample. This case is much more complex than the lightweight plaster one, because the more complicated microstructure forbids to mesh the whole microtomography image of the sample. In addition, the material is composed of 3 phases with complex shapes (see Fig. 1 (b)). 

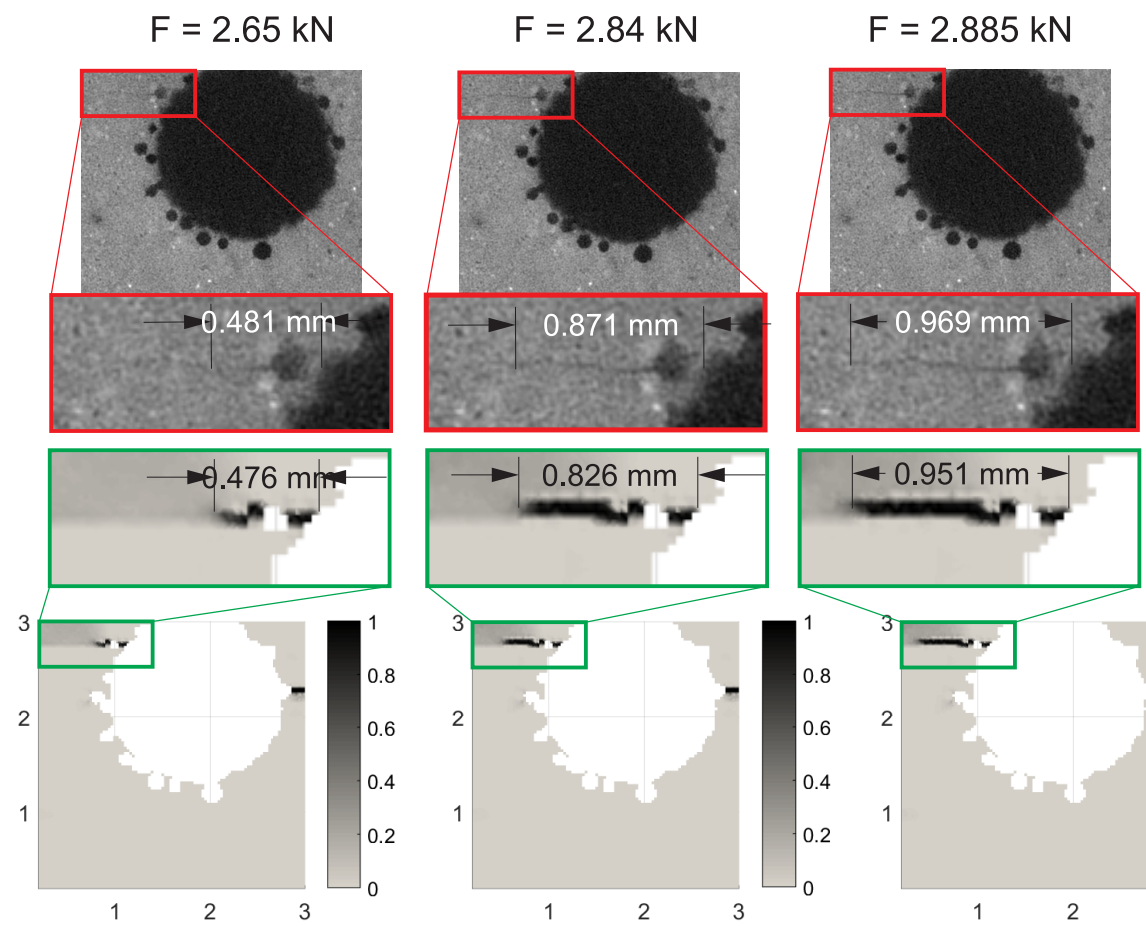

Fig. 29. Comparison between experiment (up, transformed images) and simulation (down) in terms of crack length for several loading steps, for a particular crack inside the sub-volume of the lightweight plaster sample PlasterS1.

As mentioned previously, a direct mapping of voxels properties to a regular mesh of elements from the whole 3D segmented CT-image in the simulation would lead to hundreds of millions to billions of elements which is not tractable. Then, we adopt here again the sub-volume technique described in section 6.2.1. Regarding image correlation, the lightweight concrete sample is associated with additional difficulties: it involves more badly correlated regions than in EPS plaster because of the lack of local image contrast, as in quartz sand and porosity. DVC algorithms can indeed only be run in cement paste and near interfaces. Thus, three groups, corresponding to quartz sand, cement matrix and porosity, have been distinguished among correlation points to define boundary conditions for the sub-volume method. We present an example in Fig. 30, where red colour and green colour are correlation points in quartz sand near interfaces and in cement matrix respectively. Then, for the construction of the boundary conditions at FEM nodes in-between DVC points and in addition to the general filtering and interpolations procedures described in section 5 , we require that the neighbour correlation points used for the interpolation belong to the same phase: prescribed displacements at FEM nodes in cement result from DVC points in cement only (green in Fig. 30) and those in sand grains depend on red interfacial DVC points only. Nodes in pores are removed from the mesh.

In the following, two sub-volumes are considered for a detailed comparison be- 


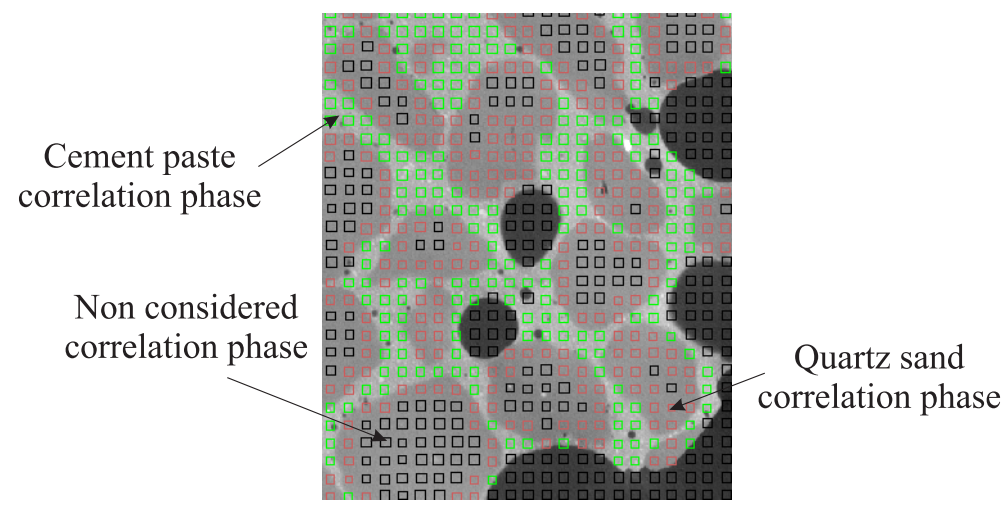

Fig. 30. Three classes of DVC points in EPS concrete. Green: Correlations point in cement paste (good accuracy); Red: Correlation points in sand near sand/cement paste interface (acceptable accuracy); Black : Points inside sand grains with insufficient contrast, not suitable for DVC. The boundary conditions in FE model result from DVC measurements at green and red points only.

tween the experimentally observed crack propagation from subtracted images and its numerical prediction by the phase-field method.

The first considered sub-volume is of size $480 \times 480 \times 72$ voxel $^{3}$ (or $4.8 \times$ $4.8 \times 0.72 \mathrm{~mm}^{3}$ ), as depicted in Fig. 31, where cracks have been observed in the experiments. A regular mesh has been used, where each 8-node element corresponds to a voxel in the segmented image, its material properties being projected on the mesh. Note that a median filter and then a watershed operator were used to extract the sand phase (inclusions). The resolution was reduced to $300 \times 300 \times 45$ to limit the computational costs. In addition, we removed all elements within the pores. The resulting mesh contains 3.6 million elements.

The material parameters of inclusions and matrix have been extracted from literature and are respectively set to: $E_{i}=60 \mathrm{GPa}, \nu_{i}=0.3, E_{m}=18 \mathrm{GPa}$, $\nu_{m}=0.2$. The toughness $g_{c}=59.3 \mathrm{~N} / \mathrm{m}$ is assumed to be identical for the different phases (following the work of Rots (1988)). The process zone length is chosen as $\ell=30 \mu \mathrm{m}$. The evolving boundary conditions obtained from DVC are applied on the boundary of the sub-volume during 300 steps, after application of both spatial and temporal interpolation and filtering operations described in section 4 . The crack propagation will be compared for several loading steps. For the sake of clarity, we do not show the matrix phase (cement paste).

The results are presented in Figs. 32 and 33 for several loading steps. For loading step $9(F=1.800 \mathrm{kN})$, we observe that the numerical crack (on the right) follows a path similar to the experimental one (on the left), but seems to be less developed.

For the loading steps $10(F=1.862 \mathrm{kN})$ and $12(F=1.937 \mathrm{kN})$, the numerical and experimental crack morphologies are remarkably similar. The main 


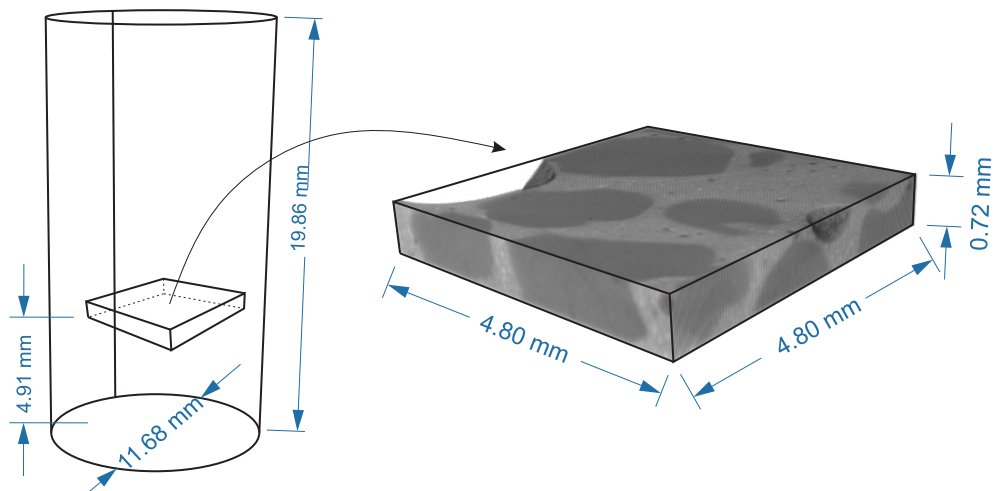

(a)

(b)

Fig. 31. Geometry of the sub-volume 1: (a) location in sample; (b) XR- $\mu \mathrm{CT}$ images of the sub-volume.
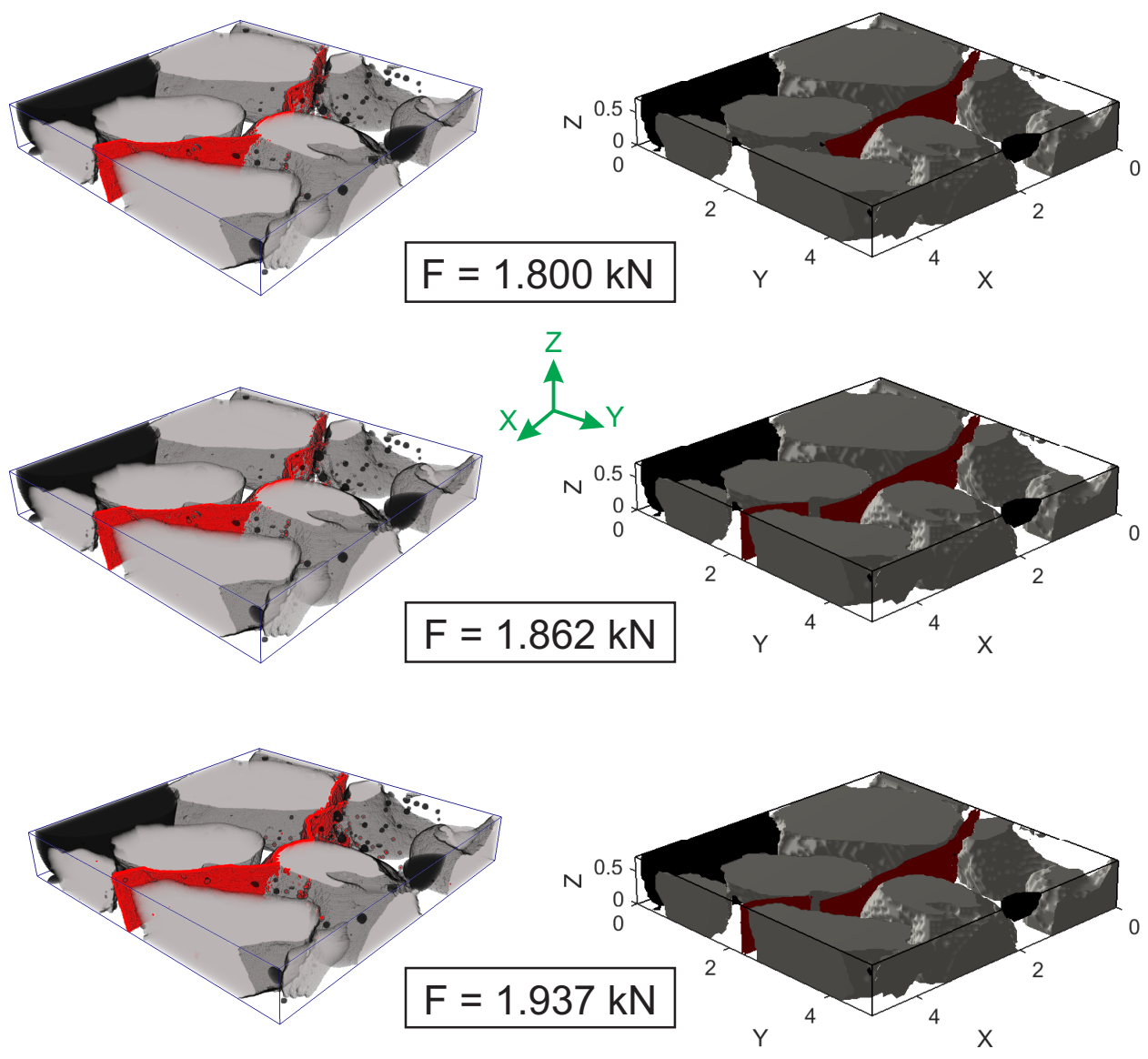

Fig. 32. Comparison between experimental crack obtained from microtomography and from phase field method of the lightweight concrete sample in sub-volume 1 for several loads, view 1 (left: experiment; right: numerical simulation.) 

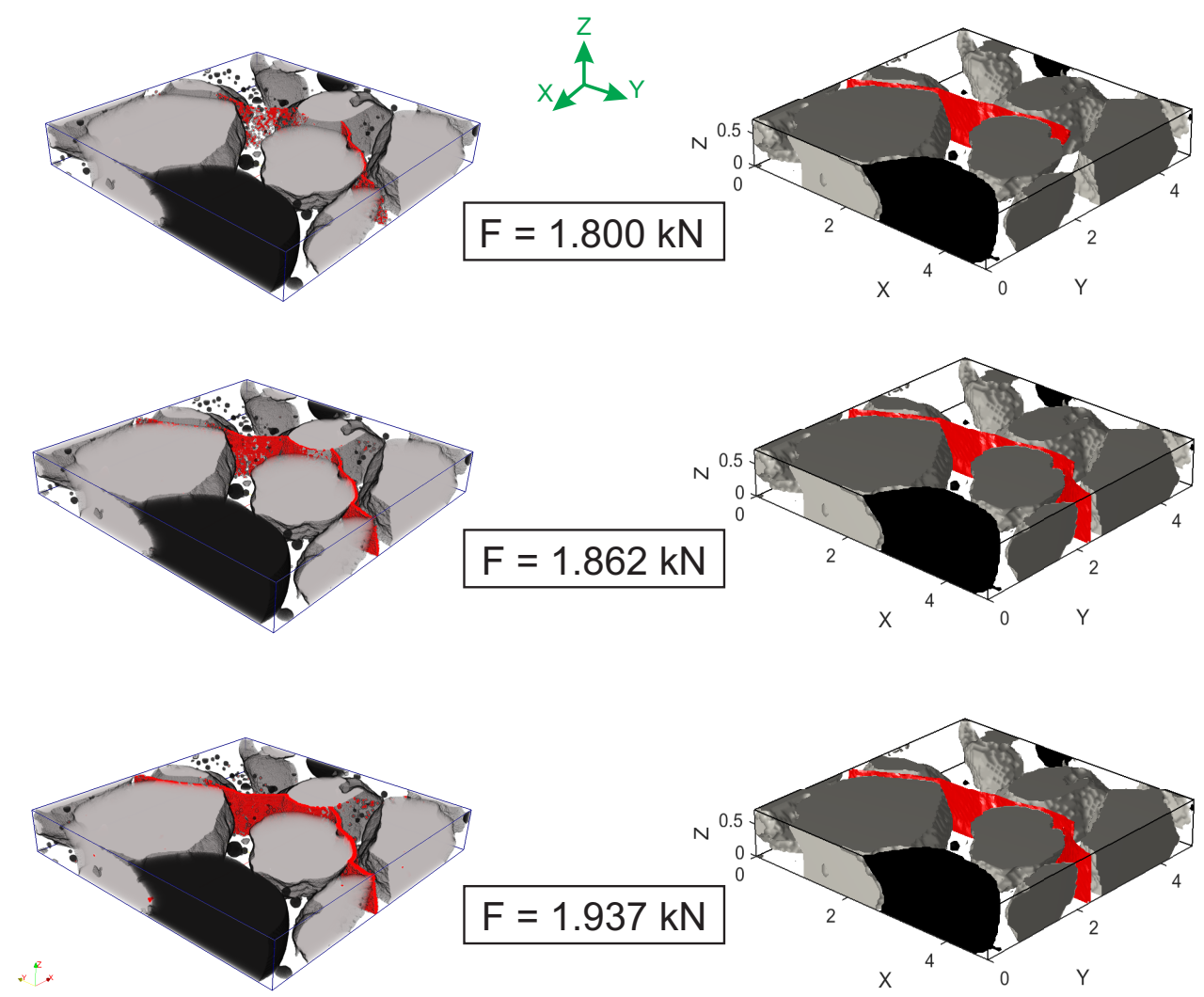

Fig. 33. Comparison between experimental crack obtained from microtomography and from phase field method of the lightweight concrete sample in sub-volume 1 for for several loads, view 2 (left: experiment; right: numerical simulation.)

discrepancies concern the crack path near the interface. Experimental observation show a crack path that often coincides with the cement paste / sand grain interface, suggesting that this interface is subjected to easier damage than the bulk cement paste matrix. Such features are not observed in the model, essentially because no interfacial damage behaviour has been taken into account so far. Extension of the phase field to interfacial cracking has been recently proposed in Nguyen et al. (2016b), but was not implemented in the present work in the 3D case. These investigations are left to further studies.

A second sub-volume is studied, of size $240 \times 240 \times 64$ voxel $^{3}$, as depicted in Fig. 34 . The obtained mesh is composed of 3.7 million of regular 8-node elements. We compare the crack propagation for experiment and numerical simulation for the loading steps 9, 10 and 12. The crack path for both simulation and experiment are compared in Figs. 35 and 36. In that case, we obtain a very good agreement regarding both crack morphology and crack length for the different loads. We believe that the agreement is better than in the first subvolume because there is less interfacial cracking in the experiment. 


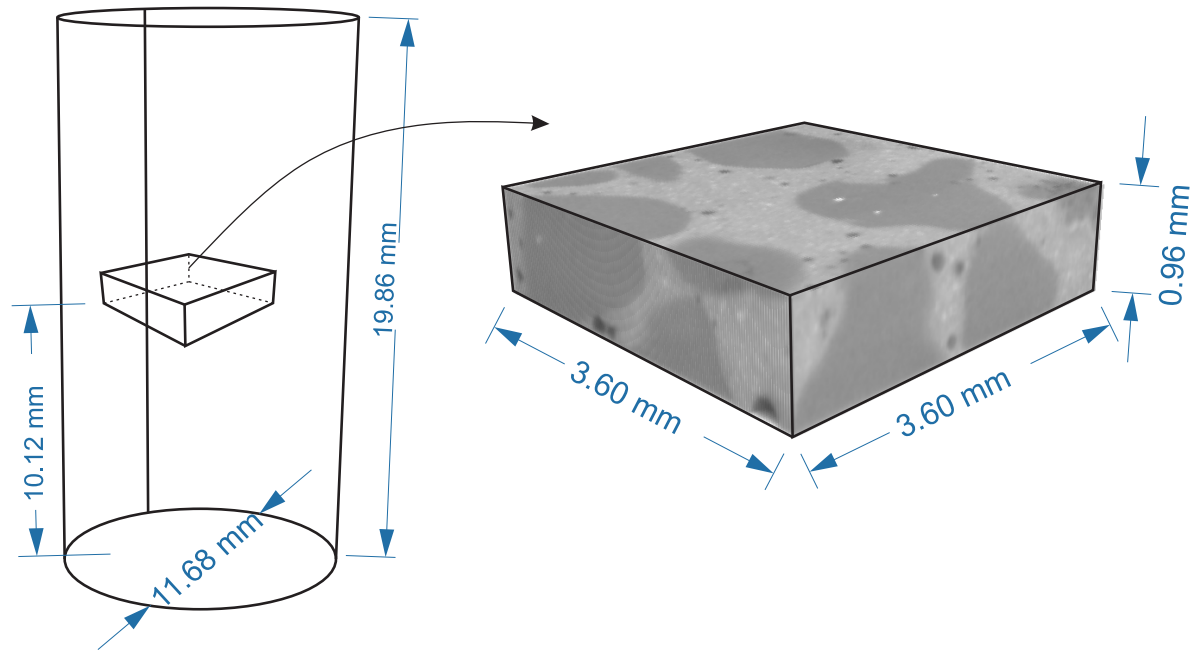

(a)

(b)

Fig. 34. Geometry of the sub-volume 2: (a) location in the sample; (b) XR- $\mu \mathrm{CT}$ images of the sub-volume.

\section{Conclusion}

In this work, we have performed the first direct comparisons, to our best knowledge, of complex 3D microcracking initiation and propagation in cementitious materials modelled by the phase field numerical method and observed in XR- $\mu \mathrm{CT}$ images recorded during in situ testing. We have focused here on lightweight plaster and concrete microstructures. Some material parameters in the damage model of the plaster matrix, including the process zone (internal) length, have been identified by an inverse approach combining experimental data and the $3 \mathrm{D}$ simulations. Analyses have been performed at both scales of the whole sample when possible (for moderately complex microstructure), and at the scale of sub-volumes in the case of very complex microstructures. In that last case, a new technique has been developed to study the microcracking at a finer scale by prescribing the displacements obtained by DVC over the boundary of a small sub-volume in the sample during the numerical simulations.

We have performed analysis on several samples with different configurations. For each sample several detailed comparisons have been performed at various loading levels, and over various 2D cuts through the 3D microstructures. The XR- $\mu \mathrm{CT}$ images of the samples before mechanical load, segmented with standard tools, have been used to define the microstructure for the numerical model. However, no assumption was made on the initiation location of the cracks and on their propagation paths: the simulations are conducted "blindly" without further input from the experiments. Images of 3D cracks at various loading levels obtained from the in situ test have been compared to the simulation results. Specific image processing routines allow to convect the crack path 

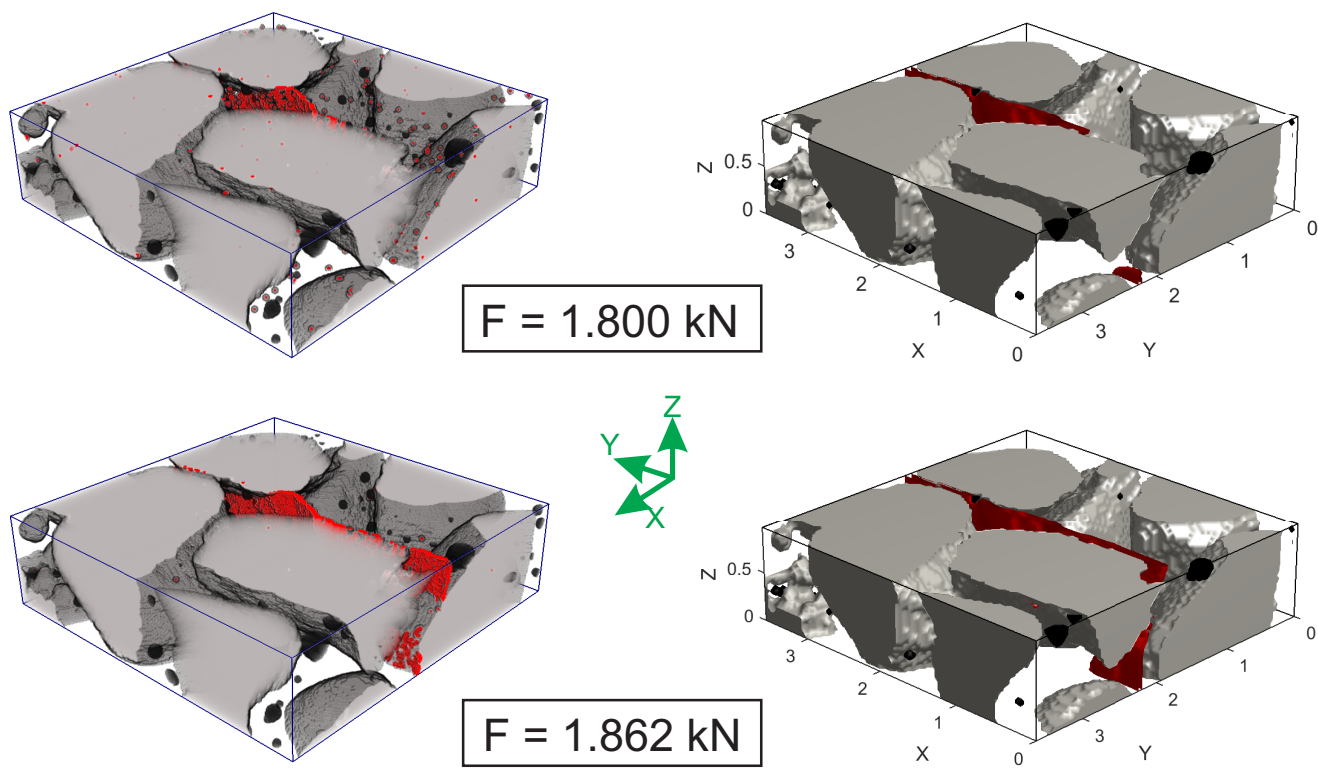

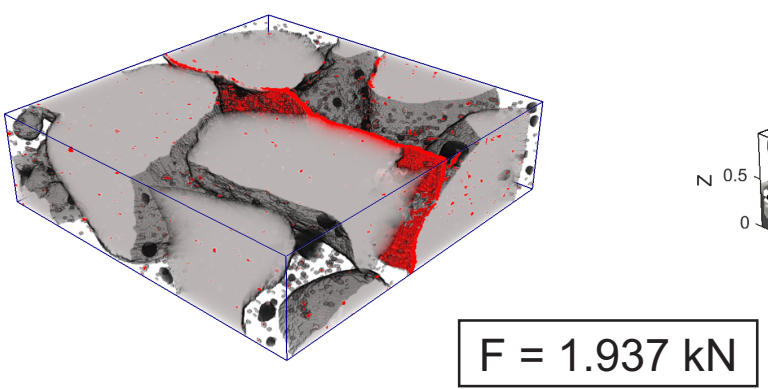

(a)

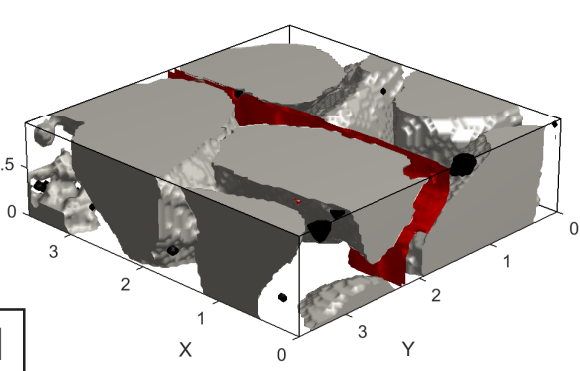

(b)

Fig. 35. Comparison between experimental crack obtained from microtomography and from phase field method of the lightweight concrete sample in sub-volume 2 for for several loads, view 1 (left: experiment; right: numerical simulation.)

in current configuration to the reference one used in the simulation, allowing a precise comparison of the morphology of the crack network. Remarkably, the 3D morphologies of micro cracking networks can be predicted accurately, as shown by many quantitative comparisons performed of some geometric features (local length and angles of cracks). A first surprising conclusion is that regularized models of quasi-brittle fracture can provide accurate predictions of real micro cracking in cementitious materials. A second one is that even though the initiation and propagation of complex networks of microcracks in heterogeneous materials made of constituent with brittle behaviour might be considered as highly stochastic in nature, it seems that the problem is actually fully deterministic when: (a) the uncertainties related to the experimental conditions (like contact conditions over the boundary of the samples) are reduced to a sufficiently small level; (b) the characteristic length of description of the microstructure geometry and thus of the induced fluctuations of the 

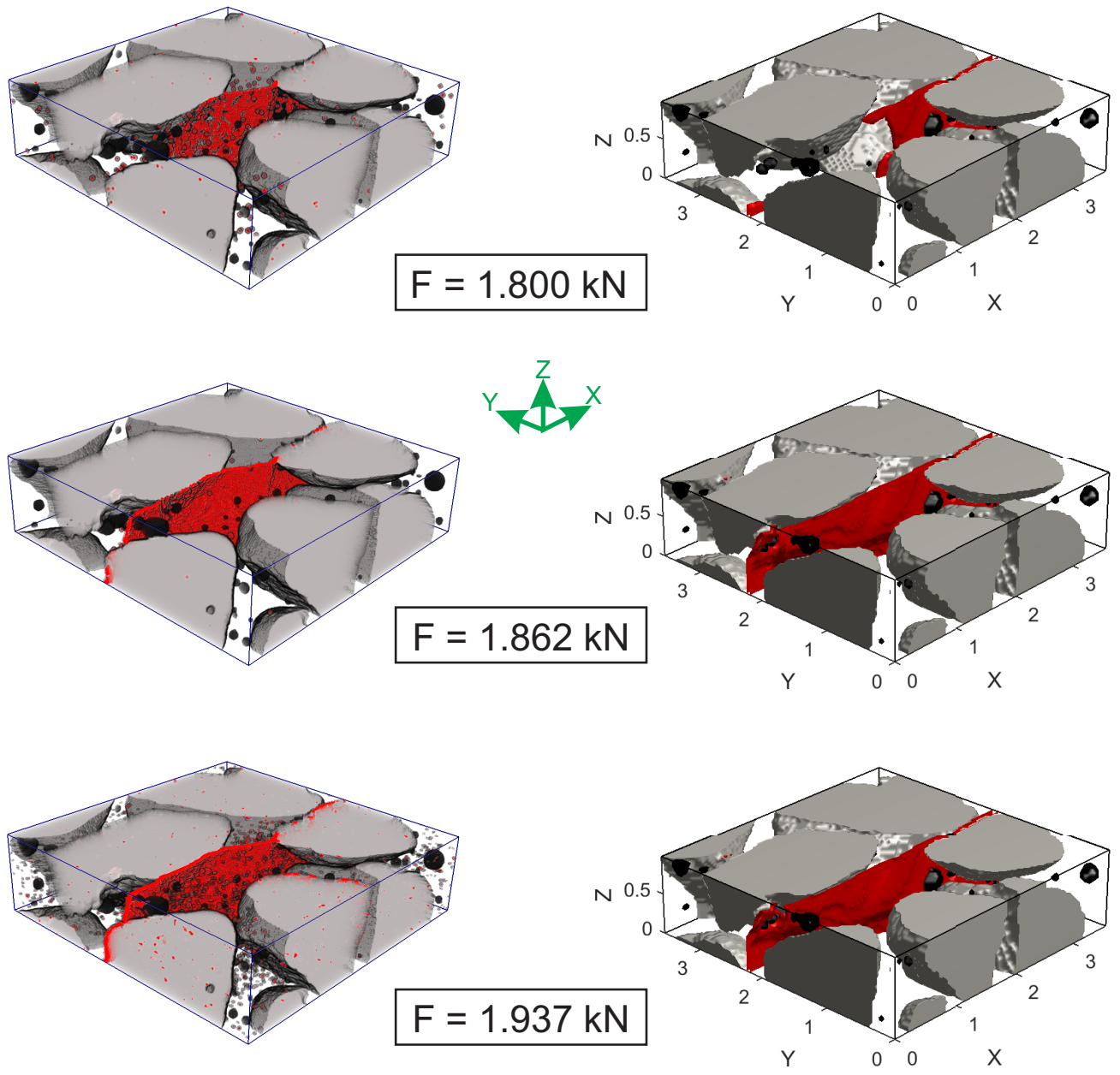

Fig. 36. Comparison between experimental crack obtained from microtomography and from phase field method of the lightweight concrete sample in sub-volume 2 for for several loads, view 2 (left: experiment; right: numerical simulation.)

local mechanical fields is lower than the process zone length of the regularized damage model of brittle fracture. Further explorations and justifications of this conjecture will have to be pursued in future studies.

\section{Acknowledgments}

This work has benefited from a French government grant managed by ANR within the frame of the national program Investments for the Future ANR-11LABX-022-01. The acquisition of the microtomography device of laboratoire Navier (ENPC) has been made possible thanks to grants from the Region 
Ile de France (SESAME 2007 program) and CNRS. The contribution of the technical team of LMS (Ecole polytechnique), and in particular V. de Greef, to the design and the manufacturing of the in situ testing device used to perform the compression tests is acknowledged, together with its funding by the "Matinex" joint program CNRS and CEA. J.Y. aknowledges the financial support from Institut Universitaire de France (IUF).

\section{References}

F. Amiot, M. Bornert, P. Doumalin, J.C. Dupré, M. Fazzini, J.J. Orteu, C. Poilâne, L. Robert, R. Rotinat, E. Toussaint, et al. Assessment of digital image correlation measurement accuracy in the ultimate error regime: main results of a collaborative benchmark. Strain, 49(6):483-496, 2013.

H. Amor, J.J. Marigo, and C. Maurini. Regularized formulation of the variational brittle fracture with unilateral contact: Numerical experiments. $J$. Mech. Phys. Solids, 57(8):1209-1229, 2009.

D.S. Babu, K.G. Babu, and W. Tiong-Huan. Effect of polystyrene aggregate size on strength and moisture migration characteristics of lightweight concrete. Cement Concrete Comp., 28(6):520-527, 2006.

B.K. Bay, T.S. Smith, D.P. Fyrhie, and M. Saad. Digital volume correlation: Three-dimensional strain mapping using x-ray tomography. Exp. Mech., 39: 217226, 1999.

A. Benallal and J.-J. Marigo. Bifurcation and stability issues in gradient theories with softening. Model. Simul. Mater. Sc., 15:S283-S295, 2007.

M. Bornert, J.M. Chaix, P. Doumalin, J.C. Dupré, T. Fournel, D. Jeulin, E. Maire, M. Moreaud, and H. Moulinec. Mesure tridimensionnelle de champs cinématiques par imagerie volumique pour lanalyse des matériaux et des structures. Inst. Mes. Metr., 3-4:4388, 2004.

M. Bornert, F. Brémand, P. Doumalin, J.-C. Dupré, M. Fazzini, M. Grédiac, F. Hild, S. Mistou, J. Molimard, J.J. Orteu, L. Robert, Y. Surrel, P. Vacher, and B. Wattrisse. Assessment of digital image correlation measurement errors: methodology and results. Exp. Mech, 49(3), 2009.

M. Bornert, F. Hild, J.J Orteu, and S. Roux. 6: digital image correlation. In Full-field measurements and identification in solid mechanics. John Wiley \& Sons, 2012.

D.P. Braides. Approximation of Free Discontinuity Problems. Springer Verlag: Berlin, 1998.

P. Coquard and R. Boistelle. Thermodynamical approach to the brittle fracture of dry plasters. J. Mater. Sci., 31(17):4573-4580, 1996.

J. Dautriat, M. Bornert, N. Gland, A. Dimanov, and J. Raphanel. Localized deformation induced by heterogeneities in porous carbonate analysed by multi-scale digital image correlation. Tectonophysics, 503(1):100-116, 2011.

L.A. Feldkamp, L.C.Davis, and J.W.Kress. Practical cone-beam algorithm. 
Constr. Build. Mater., 1:612619, 1984.

E. Ferrié, J.-Y. Buffière, and W. Ludwig. 3D characterisation of the nucleation of a short fatigue crack at a pore in a cast $\mathrm{Al}$ alloy using high resolution synchrotron microtomography. Int. J. Fatigue, 27:1215-1220, 2005.

G.A. Francfort and J.J. Marigo. Revisiting brittle fracture as an energy minimization problem. J. Mech. Phys. Solids, 46(8):1319-1342, 1998.

D. Grégoire, H. Maigre, J. Réthoré, and A. Combescure. Dynamic crack propagation under mixed-mode loading comparison between experiments and X-FEM simulations. Int. J. Solids Struct., 44:6517-6534, 2007.

E. Heripre, M. Dexet, J. Crepin, L. Gélebart, A. Roos, M. Bornert, and D. Caldemaison. Coupling between experimental measurements and polycrystal finite element calculations for micromechanical study of metallic materials. Int. J. Plasticity, 23(9):1512-1539, 2007.

S. Ismonov and S.R. Daniewicz. Simulation and comparison of several crack closure assessment methodologies using three-dimensional finite element analysis. Int. J. Fatigue, 32:1322-1329, 2010.

R.P. Janeiro and H.H. Einstein. Experimental study of the cracking behavior of specimens containing inclusions (under uniaxial compression). Int. J. Fract., 164(1):83-102, 2010.

C. Kuhn, A. Schlueter, and R. Müller. A phase-field description of dynamic brittle fracture. Comput. Mater. Sc., 108:374-384, 2015.

J. Lachambre, J. Réthoré, A. Weck, J.-Y. Buffière, A. Combescure, F. Hild, and S. Roux. Extraction of stress intensity factors for 3D small fatigue cracks using digital volume correlation and X-ray tomography. Int. J. Fatigue, 71: 3-10, 2015.

F. Lagier, X. Jourdain, C. De Sa, F. Benboudjema, and J.B. Colliat. Numerical strategies for prediction of drying cracks in heterogeneous materials: Comparison upon experimental results. Eng. Struct., 33:920-931, 2011.

C. Landron, E. Maire, O. Bouaziz, J. Adrien, and L. Lecarme. Damage quantification in aluminium alloys using in situ tensile tests in X-ray tomography. Acta Mater., 59(20), 2011.

N. Lenoir, M. Bornert, J. Desrues, P. Bésuelle, and G. Viggiani. Volumetric digital image correlation applied to x-ray microtomography images from triaxial compression tests on argillaceous rock. Rock. Strain, 43(3):193205, 2007.

N. Liu and B. Chen. Experimental study of the influence of EPS particle size on the mechanical properties of EPS lightweight concrete. Constr. Build. Mater., 68:227-232, 2014.

S. Lu, E.N. Landis, and D.T. Keane. X-ray microtomographic studies of pore structure and permeability in portland cement concrete. Mater. Struct., 39 (6):611-620, 2006.

E. Maire, S.X. Zhou, J. Adrien, and M. Di Michiel. Damage quantification in aluminium alloys using in situ tensile tests in X-ray tomography. Eng. Frac. Mech., 78(15), 2011.

S. Meille, M. Saâdaoui, P. Reynaud, and G. Fantozzi. Mechanisms of crack 
propagation in dry plaster. J. Eur Ceram. Soc., 23(16):3105-3112, 2003.

C. Miehe, M. Hofacker, and F. Welschinger. A phase field model for rateindependent crack propagation: Robust algorithmic implementation based on operator splits. Comput. Meth. Appl. Mech. Eng., 199:2776-2778, 2010.

K. Miled, R. Le Roy, K. Sab, and C. Boulay. Compressive behavior of an idealized eps lightweight concrete: size effects and failure mode. Mech. Mater., 36(11):1031-1046, 2004.

K. Miled, K. Sab, and K. Le Roy. Particle size effect on EPS lightweight concrete compressive strength: experimental investigation and modelling. Mech. Mater., 39(3):222-240, 2007.

N. Moës, J. Dolbow, and T. Belytschko. A finite element method for crack growth without remeshing. Int. J. Numer. Meth. Eng., 46(1):131-150, 1999.

T.T. Nguyen. Modeling of complex microcracking in cement based materials by combining numerical simulations based on a phase-field method and experimental 3D imaging. PhD thesis, Université Paris-Est, 2015.

TT. Nguyen, J. Yvonnet, Q-Z. Zhu, M. Bornert, and C. Chateau. A phase field method to simulate crack nucleation and propagation in strongly heterogeneous materials from direct imaging of their microstructure. Eng. Fract. Mech., 139:18-39, 2015.

T.T. Nguyen, J. Yvonnet, M. Bornert, C. Chateau, K. Sab, R. Romani, and R. Le Roy. On the choice of parameters in the phase field method for simulating crack initiation with experimental validation. Int. J. Fract., 197 (2):213-226, 2016a.

T.T. Nguyen, J. Yvonnet, Q.-Z. Zhu, M. Bornert, and C. Chateau. A phasefield method for computational modeling of interfacial damage interacting with crack propagation in realistic microstructures obtained by microtomography. Comput. Meth. Appl. Mech. Eng., 2016b.

O. Nouailletas, C. La Borderie, C. Perlot, P. Rivard, and G. Ballivy. Experimental study of crack closure on heterogeneous quasi-brittle material. $J$. Eng. Mech., 141(11), 2015.

K. Oguni, T. Okinaka M.L.L. Wijerathne and, and M. Hori. Crack propagation analysis using pds-fem and comparison with fracture experiment. Mech. Mater., 41:1242-1252, 2009.

J. Rannou, N. Limodin, J. Réthoré, A. Gravouil, W. Ludwig, M.-C. BaiettoDubourg, J.-Y. Buffière, A. Combescure, F. Hild, and S. Roux. Three dimensional experimental and numerical multiscale analysis of a fatigue crack. Comput. Meth. Appl. Mech. Eng., 199:1307-1325, 2010.

$\mathrm{R}$. Romani. Rupture en compression des structures hétérogènes à base de matériaux quasi-fragiles. PhD thesis, Paris 6, 2013.

R. Romani, M. Bornert, D. Leguillon, R. Le Roy, and K. Sab. Detection of crack onset in double cleavage drilled specimens of plaster under compression by digital image correlation-Theoretical predictions based on a coupled criterion. Eur. J. Mech. A-Solids, 51:172-182, 2015.

J.G. Rots. Computational modeling of concrete fracture. PhD thesis, Technische Hogeschool Delft, 1988. 
C. Roux-Langlois, A. Gravouil, M.-C. Baietto, J. Réthoré, F. Mathieu, F. Hild, and S. Roux. DIC identification and X-FEM simulation of fatigue crack growth based on the Williams series. Int. J. Solids Struct., 53:38-47, 2015.

M. Saadaoui, P. Reynaud, G. Fantozzi, F. Peronnet, and J.P. Caspar. Slow crack growth study of plaster using the double torsion method. Ceram. Int., 26(4):435-439, 2000.

J. Schindelin, I. Arganda-Carreras, and E. Frise. Fiji: an open-source platform for biological-image analysis. Nature methods, 9(7):676680, 2012.

H.W. Schreier, J.R. Braasch, and M.A. Sutton. Systematic errors in digital image correlation caused by intensity interpolation. Opt. Eng., 39, 2000.

E. Soppa, P. Doumalin, P. Binkele, T. Wiesendanger, M. Bornert, and S. Schmauder. xperimental and numerical characterisation of in plane deformation in two-phase materials. Comp. Mat. Sci., 21, 2001.

T.S. Sprague. X-ray tomography for evaluation of damage in concrete bond. PhD thesis, University of Washington, 2006.

H. Toda, I. Sinclair, J.-Y. Buffière, E. Maire, K.H. Khor, P. Gregson, and T. Kobayashi. A 3D measurement procedure for internal local crack driving forces via synchrotron X-ray microtomography. Acta Mater., 52:1305-1317, 2004.

U. Toshiyuki, L. Helfen, and T. Morgeneyer. In situ laminography study of three-dimensional individual void shape evolution at crack initiation and comparison with Gurson-Tvergaard-Needleman-type simulations. Acta Mater., 78:254270, 2014.

G. Vekinis, M.F. Ashby, and P.W.R. Beaumont. Mechanisms of crack propagation in dry plaster. J. Mater. Sci., 28(12):3105-3112, 1993.

R.H.C. Wong, P. Lin, and C.A. Tang. Experimental and numerical study on splitting failure of brittle solids containing single pore under uniaxial compression. Mech. Mater., 38:142-159, 2006.

A. Yin, X. Yang, G. Zeng, and H. Gao. Fracture simulation of pre-cracked heterogeneous asphalt mixture beam with movable three-point bending load. Constr. Build. Mater., 65:232242, 2014.

A. Yin, X. Yang, C. Zhang, G. Zeng, and Z. Yang. Three-dimensional heterogeneous fracture simulation of asphalt mixture under uniaxial tension with cohesive crack model. Constr. Build. Mater., 76:103-117, 2015. 